\title{
Thick disk and old disk carbon-rich giants in the Sun vicinity ${ }^{\star, \star \star}$
}

\author{
J. Bergeat, A. Knapik, and B. Rutily \\ Centre de Recherche Astronomique de Lyon (UMR 5574 du CNRS), Observatoire de Lyon, \\ 9 avenue Charles André, 69561 St-Genis-Laval Cedex, France
}

Received 7 June 2001 / Accepted 7 January 2002

\begin{abstract}
Making use of the HIPPARCOS data and refining a previous study of the space distribution of the carbon-rich giant stars located in the vicinity of the Sun (Paper I), we fully investigate their space distributions and space velocities on the basis of our photometric grouping (CVi i.e. carbon variable stars with $i=1$ to 7 ; $\mathrm{HC} j$ i.e. hot carbon stars with $j=0$ to 5). As expected, the $\mathrm{CH}$ stars (a subset of the HC stars delineated on the grounds of spectroscopic criteria) need to be considered separately. We also used groupings according to variability classes. The various biases affecting the use of the data are taken into account as far as possible. The mean distance to the Galactic plane of the faint $\left(\left\langle M_{\mathrm{bol}}\right\rangle \geq-3.5\right)$ HC-stars amounts to $0.5 \mathrm{kpc}$ compared to $0.15 \mathrm{kpc}$ for the bright $\left(\left\langle M_{\mathrm{bol}}\right\rangle \leq-3.5\right) \mathrm{CV}$-stars. Exponentially decreasing distributions are fit with distance scales of $0.95 \pm 0.06 \mathrm{kpc}$ and $0.19 \mathrm{kpc}$ respectively, and a normalization factor of $5.2 \%$ to $7.3 \%$ for the former component, compatible with a thick disk and thin disk respectively. Projected surface densities on the Plane are given with a total of about $76 \mathrm{kpc}^{-2}$, including $6 \%$ of $\mathrm{CH}$ stars and at least $18 \%$ for the other HC-stars (namely the HC'-sample). While halo-type velocities are found for $\mathrm{CH}$ stars with a substantial drift of $-112 \mathrm{~km} \mathrm{~s}^{-1}$, the solar reflex velocities and residual-velocity dispersions for the HC'-sample are about twice those of the CV-sample, close to thick disk and thin disk values respectively. In summary, we identify the HC-sample as a component of the thick disk contaminated by the $\mathrm{CH}$ stars which are a spheroidal contribution, and possibly by CV-stars at

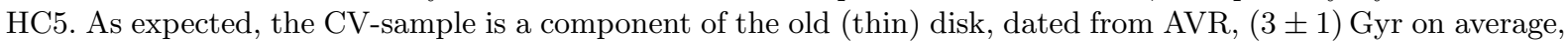
but with a likely spread from a few $10^{8} \mathrm{Myr}$ up to 8-12 Gyr. While the former (HC) represents very old low mass stars (initial masses less than $1.15 M_{\odot}$ but subject to mass-loss), the latter (CV) are younger stars with higher initial masses on average (up to a few solar masses). The high frequency of HC'-stars rules out models requiring rare events. Better modeling of mixing events in low mass stars on the RGB and AGB could help. Less dragged up carbon is needed to transform the low-mass stars with a low $\mathrm{O} / \mathrm{H}$ ratio into carbon giants.
\end{abstract}

Key words. stars: AGB and post-AGB - stars: carbon - stars: late-type - stars: variables: general stars: kinematics

\section{Introduction}

The carbon-rich giant stars occupy a special place in the realm of stellar evolution. They are stars of low and intermediate initial masses evolved far from the main sequence up to the red giant branch and asymptotic branch where they experience thermal pulses (TPAGB; Wallerstein \& Knapp 1998; Busso et al. 1999). Alternatively, anomalous surface abundances may result from mass exchange in a binary system, with a TP-AGB companion that became a white dwarf (Han et al. 1995 and references therein). This is the model accepted for BaII stars, many of them

\footnotetext{
Send offprint requests to: J. Bergeat, e-mail: jb@obs.univ-lyon1.fr

* This research has made use of the Simbad database operated at CDS, Strasbourg, France.

$\star \star$ Partially based on data from the ESA HIPPARCOS astrometry satellite.
}

having proved to be binary members (Mc Clure et al. 1980; Jorissen \& Boffin 1992). The low-luminosity carbon stars classified into the R-types however fail to show any evidence of binarity, and Mc Clure (1997a) considered they could be the product of coalescence of components.

With the existence of carbon dwarfs (Dahn et al. 1977; Green et al. 1991; Bothun et al. 1991), some carbon giants may be evolved from carbon dwarfs, exactly like dwarf BaII stars (e.g. Bergeat \& Knapik 1997) that are potential precursors for some future BaII subgiants and giants. Those carbon dwarfs are likely members of binary systems (Liebert et al. 1994; Green \& Margon 1994) and their velocities of several hundreds of $\mathrm{km} \mathrm{s}^{-1}$ relative to the Sun are typical of a spheroidal population (halo).

We investigate the space distributions and space velocities from HIPPARCOS data, of a sample of about 300 carbon giants first analyzed by Knapik et al. (1998, hereafter Paper I). They proposed a model of the distribution of 
observed parallaxes, making use of the cumulative distribution of HIPPARCOS proper motions which are very accurate when compared with those previously available. The HIPPARCOS carbon stars are close to the Sun. They only show some concentration towards the Galactic plane while the 5987 stars in Stephenson's (1989) catalogue exhibit, in addition, a positive galactocentric gradient towards Galactic anticenter (Fuenmayor 1981), and clumps correlated with the local spiral arms (Cygnus-Perseus and Sagittarius-Carinae: Stephenson's Fig. 2). Having described and taken into account the biases which affect the mean values from HIPPARCOS data, Knapik et al. (Paper I) adopted a flat subsystem or slab. They found their data to be roughly consistent with uniform density in star number. Part of the present paper is intended to refine this picture, especially concerning the frequency distribution vs. height (i.e. distance to galactic plane $|z|)$.

Classical analyses of velocity data include the determination of the solar reflex velocity, and then the evaluation of the maximal dispersions along the three ellipsoid axes (see e.g. Delhaye 1965 for a summary of early results). They make use of radial velocities and/or proper motions. A better constraint of the kinematic solution is obtained when individual parallaxes are available (Mihalas \& Binney 1981, Sects. 6 and 7). For carbon stars, it was impracticable before HIPPARCOS since no reliable parallaxes were available.

The early studies on late-type giants are summarized in Blanco (1965) and on variables by Plaut (1965). The solar motions and velocity ellipsoids for early $\mathrm{R}$ stars (Vandervort 1958; Mc Leod 1947) seem similar to those of $\mathrm{G}$ and $\mathrm{K}$ dwarfs. The most extensive study was by Dean (1976), which included 427 stars, mainly classified N or late-R. The author concluded that the obtained kinematic solution is close to the one obtained for F5 dwarfs. Among the R stars, the CH stars (Keenan 1942; Bidelman 1956) are on average high velocity stars with respect to the Sun. They are believed to be members of binary systems (McClure 1997b) but the eventual orbital velocities should be small on average $\left(0-15 \mathrm{~km} \mathrm{~s}^{-1}\right)$ when compared to the high velocities observed with respect to the Sun. Hartwick \& Cowley (1985) studied 51 confirmed and probable CH stars. They concluded that they are halo tracers. A majority of carbon giants and related objects are variables of various types in the General Catalogue of Variable Stars (GCVS, Kholopov et al. 1985), namely Lb (irregulars), SR (semi-regulars a or b) and M (Miras). The oxygen-rich long period variables (LPVs) show specific kinematic solutions with large solar reflex velocities and larger dispersions at shorter periods (e.g. Delhaye 1965; Mihalas \& Binney 1981). A question is whether the carbon-rich LPVs display a similar behavior.

Apart from the $\mathrm{CH}$ stars which are associated with the spheroidal component, the carbon giants are mainly disk objects. It is especially interesting to explore the connection of those stars with the old (thin) disk and with the recently discovered thick disk (e.g. Freeman 1987a). These structures deserve their many recent studies (e.g.
Buser et al. 1999; Schwarzkopf \& Dettmar 2000; Chiba \& Beers 2000; Prochaska et al. 2000; Kerber et al. 2001; Ojha 2001).

The first classification of the carbon-rich giants in discrete photometric groups (13 groups named $\mathrm{HC} i$ and $\mathrm{CV} j$ with $i=0$ to 5 and $j=1$ to 7 , and the fourteenth SCV as a transition to S stars), was established by Knapik \& Bergeat (1997), Bergeat et al. (1999) and Knapik et al. (1999). The new effective temperatures proposed for carbon-rich giants and related objects by Bergeat et al. (2001) rely on spectral energy distributions (SEDs), model atmospheres and measured angular diameters. The classification and the homogeneous temperature scale obtained proved to be tightly correlated. It was concluded that the main parameter of this photometric classification is effective temperature (Bergeat et al. 2001). This is the first classification to achieve this, providing a sound basis for further model atmosphere analysis. A good correlation is also found with luminosities (Knapik et al. 2001 and Table 3 hereafter). Sampling according to our photometric classification is thus preferred to that making use of spectral classification, even in its refreshed version (Keenan 1993).

The statistical biases affecting parallaxes and deduced quantities were studied in Paper I. They are re-examined in Sect. 2 with special attention paid to differences between the $\mathrm{HC}$ and $\mathrm{CV}$-groups. The mean distances $\langle|z|\rangle$ to the Galactic plane are investigated in Sect. 3, while the influence of the Malmquist bias is estimated in Sect. 4 . After correction for selection effects in sampled cylinders, the distributions of $\mathrm{HC}$ - and CV-stars with distances $|z|$ to the Plane are investigated in Sect. 5, and local space densities derived. The projected surface densities of carbon giants according to photometric groups (Sect. 6) and effective temperatures (Sect. 7) are presented. A first crude analysis of kinematic data is then performed, making use of mean radial and tangential velocities with respect to the Sun (Sect. 8). Finally the reflex solar velocities (Sect. 9) and stellar residual-velocity ellipsoids (Sect. 11) are derived, the former for variability classes as well (Sect. 10). The results are discussed (Sect. 12) in terms of two distinct populations: the CV-stars associated with the old (thin) disk and most of the HC-stars (HC'-sample, i.e. non-CH HC-stars) with the thick disk. The $\mathrm{CH}$ stars are confirmed as a halo component.

\section{The statistical biases}

\subsection{The distribution of true parallaxes}

Knapik et al. (Paper I) studied the space distribution of the nearly 300 carbon-rich giant stars observed by the satellite HIPPARCOS (ESA 1997). No appreciable correlation with interstellar extinction, galactocentric distance and/or spiral arms structures was detected in this sample of nearby stars (Sect. 2 of Paper I). Due to the limited accuracy of the observed parallaxes $\varpi_{0}$, the statistical use of parallaxes, distances, absolute magnitudes etc. 
is hampered by statistical biases. The bias investigated by Lutz \& Kelker (1973, hereafter LK) is twofold

- the effect of the true spatial distribution of stars on parallaxes measurements for a given standard deviation (which amounts to zero only for uniformlydistributed true parallaxes), and

- the non-Gaussian distribution of quantities which are non-linear functions of parallaxes (such as distances or absolute magnitudes), even for a Gaussian distribution of parallaxes (Smith \& Eichhorn 1996), with the additional effect of truncation, at least of negative observed parallaxes.

Even in the hypothetic case of vanishingly small errors on observations (perfect measurements) resulting in negligible influence of those biases, the Malmquist bias (Malmquist 1924, 1936) is present since the employed sample was selected on the basis of a limiting blue or visual apparent magnitude. The effects of those biases having been taken into account, the true spatial distribution can be obtained. In Paper I, uniform sliced cylinders (slabs) with axes normal to the Plane were adopted.

The relative accuracies of the HIPPARCOS proper motions are far higher than those of the corresponding observed parallaxes in the catalogue (ESA 1997). In addition, no appreciable bias is expected on the former data. Adopting a model by Hanson (1979) on the cumulative distribution of proper motions, Knapik et al. (Paper I) established a simple power law

$P(\varpi) \propto \varpi^{-(2.35 \pm 0.10)}$

for the distribution fonction of true parallaxes in the whole sample of the carbon-rich stars in the HIPPARCOS Catalogue. They modeled the distributions of the observed parallaxes for three distinct error ranges (their Fig. 2), and calculated for statistical purposes the expected true parallaxes of the parent population of the sample (their Sect. 5). The good correlation (their Fig. 3b) found with the relative photometric angular diameters (independent data) confirmed the validity of the approach.

\subsection{The HC-subsample}

The hot carbon stars (HC-stars; Bergeat et al. 1999) appear less concentrated toward the Galactic plane (Paper I) than the cool carbon variables (CV-stars; Knapik \& Bergeat 1997 and Knapik et al. 1999). This assumption based on galactic latitudes (Stephenson 1989) was confirmed by the HIPPARCOS data. Since the HIPPARCOS sample and Paper I model are dominated by about 195 CV-stars, we focus here on the case of the subsample of 104 HC-stars, repeating the analysis of the cumulative distribution of proper motions (Sect. 3 of Paper I). This more dispersed diagram led us to an exponent of $-2.25 \pm 0.15$, which is not significantly different from that in Eq. (1). Finally, we adopt the latter for the whole sample of carbon stars, including peculiar objects.
The distribution of Eq. (1) results from the Malmquist bias on the magnitude-limited HIPPARCOS sample. The limiting visual apparent magnitude $V_{1}^{\prime} \simeq 10.0 \pm 0.50$ was adopted in Paper I for the whole sample, leading to the absolute visual magnitudes

$M_{V l}=V_{1}^{\prime}-10.0+5 \log \varpi \simeq 5 \log \varpi$.

The distributions in dereddened magnitudes of the HCand CV-groups both look nearly Gaussian in shape, but with a slight difference in limiting magnitudes, namely

$\left(V_{\mathrm{l}}^{\prime}\right)_{\mathrm{HC}} \simeq 10.44 \pm 0.20$

for the HC-stars and, for the CV-stars,

$\left(V_{1}^{\prime}\right)_{\mathrm{CV}} \simeq 9.57 \pm 0.20$.

Their maximas are located at $\left(V_{0}\right)_{\max } \simeq 9.50 \pm 0.20$ and $\left(V_{0}\right)_{\max } \simeq 8.35 \pm 0.20$ respectively. Corrections to the effect of the Malmquist bias on mean absolute magnitudes are proposed in Sect. 4.

\subsection{A global model of the influence of the Malmquist bias}

Making use of Eq. (2) for the whole sample, the distribution

$N\left(M_{V}\right) \simeq 50.68 \exp \left[-\left(M_{V}+1.5\right)^{2} / 2(1.06)^{2}\right]$

was adopted in Paper I. For the Malmquist bias, the correction factor $(f \geq 1)$ on the number density was calculated as

$f(\varpi)=\left[\int_{-\infty}^{x_{1}}(2 \pi)^{-1 / 2} \exp \left(-x^{2} / 2\right) \mathrm{d} x\right]^{-1}$.

Equation (12) in Paper I was however erroneously written with a factor $2^{-1 / 2}$. The correct version is thus

$x_{1}=\left(M_{V 1}+1.5\right) / 1.06 \simeq 1.412+4.708 \log \varpi$

with Eq. (13) in Paper I being replaced by

$f(\varpi)=f(1) \varpi^{-m} \simeq 1.08 \varpi^{-m}$.

Then the exponent $m \simeq 0.70 \pm 0.07$ is a good fit on the $0.5 \leq \varpi \leq 1.2$ mas interval where $65.6 \%$ of our stars are located. We obtained $f \leq 1.035$ for stars with $\varpi \geq 1.2(31.4 \%)$. The statement in Paper I however remains unchanged, that is "for a remaining $3 \%$ of stars at $\varpi \leq 0.5$, the $f$-factor is underestimated of typically $15 \%$ when calculated from Eq. (13)", i.e. from Eq. (8) in the present paper. Finally, only slight changes occur in the exponent (0.70 instead of 0.67 ) and intervals (the above 1.2 mas replaces a former 1.4 mas value). The individual values are given in Table 1 . Very similar results $(m \simeq 0.63)$ were derived from an assumed rectangular distribution of absolute magnitudes $M_{V}$ (Sect. 7.3 of Paper I). We finally adopt the exponent $m \simeq 0.67 \pm 0.07$. 
Table 1. The correction factor $(f)$ of the Malmquist bias as a function of the true parallax $\varpi$ for a well-documented subsample of 262 HIPPARCOS carbon stars. The slope change becomes apparent near $\varpi \simeq 1.0$ mas.

\begin{tabular}{c|cccccccccccccc}
\hline$\varpi$ & 0.35 & 0.40 & 0.45 & 0.50 & 0.55 & 0.60 & 0.70 & 0.80 & 0.90 & 1.0 & 1.122 & 1.259 & 1.50 & 2.00 \\
$f(\varpi)$ & 4.321 & 3.103 & 2.423 & 2.000 & 1.738 & 1.554 & 1.329 & 1.204 & 1.131 & 1.086 & 1.052 & 1.031 & 1.013 & 1.0023 \\
\hline
\end{tabular}

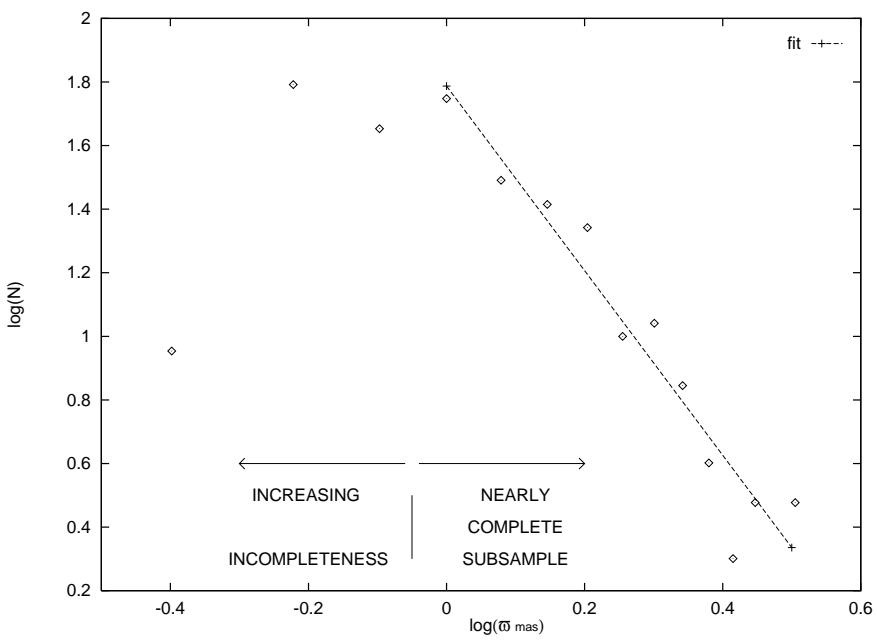

Fig. 1. The distribution of the HIPPARCOS carbon stars as a function of the estimated true parallax. Power law (10) is shown as a dashed line. Beyond $1 \mathrm{kpc}$, the increasing influence of incompleteness of the sample is clearly seen.

As stated in Paper I, roughly correcting the distribution of Eq. (1), we obtain

$N(\varpi) \propto \varpi^{-q} \mathrm{~d} \varpi$

with $q \simeq 2.35+0.67 \simeq 3.02 \pm 0.12$, for the space distribution of carbon giants in the Sun vicinity. The distribution $N(\varpi)$ obtained before correction is shown in Fig. 1. Two portions may be distinguished viz.

$-\varpi \geq 1$ mas, fitted with

$\log N(\varpi) \simeq-(2.90 \pm 0.27) \log \varpi+(1.79 \pm 0.13)$

that is an exponent amounting to the above-quoted q-value within errors and,

$-\varpi \leq 1 \mathrm{mas}$, where the distribution goes through a maximum before decreasing rapidly.

The power law $(-2.35)$ in Eq. (1) is a global result for the HIPPARCOS Malmquist-biased sample. An unbiased sample would exhibit $q \simeq 3$ which was interpreted in Paper I in terms of a uniformly-populated slab. The distributions of the CV-and HC-samples displayed slopes similar to that of Eq. (10) for Fig. 1 data. The above transition at $\varpi_{\mathrm{t}} \simeq 1$ mas however results from $\left(\varpi_{\mathrm{t}}\right)_{\mathrm{HC}} \simeq 0.6$ mas for the HC-stars combined with $\left(\varpi_{\mathrm{t}}\right)_{\mathrm{CV}} \simeq 1.2$ mas for the CV-stars. This is a direct consequence of the more pronounced concentration of the latter objects toward the Plane. Future space missions like GAIA (ESA 1995) will allow more complete sampling, reaching the edge of the Galactic disk and a much better accuracy, reducing the Malmquist and Lutz-Kelker biases to unprecedented low levels. Then, the main difficulty will probably come from the brightness distributions on the discs of those large angular diameter objects.

\section{The mean distances $\langle|z|\rangle$ to the Galactic plane}

Following the analysis of the HIPPARCOS data on carbon stars, a simplified model of a uniformly-populated slab or flat cylinder was proposed in Sect. 8 of Paper I. Its theoretical distribution was

$N(\varpi) \mathrm{d} \varpi \propto 2 \pi h \varpi^{-3} \mathrm{~d} \varpi$

with an exponent amounting to exactly 3 . The $h$ parameter is the cylinder height, exploring the $\pm h / 2$ interval centered on the Galactic plane. This disk of radius about $3 \mathrm{kpc}$ was introduced to model the space distribution of about 300 HIPPARCOS carbon stars. It was stated in Paper I that $h \simeq 0.6-0.8 \mathrm{kpc}$ should be adopted for $\mathrm{N}$ stars (mainly CV-stars) while $h \simeq 1.1-1.3 \mathrm{kpc}$ can fit the distribution of the R-stars (essentially HC-stars). This is the crude model we intend to refine hereafter.

\subsection{The mean distances and their associated bias}

The true parallaxes as estimated in Sect. 5 of Paper I can, at least to a first approximation, be used to derive mean parallaxes $(\langle\varpi\rangle)$ free of the Lutz \& Kelker (1973) bias. The pdf of the "observed" distance (as compared to the "true" distance $D$ ) is

$f\left(D_{0}\right)=\exp \left[-\left(1 / D_{0}-1 / D\right)^{2} / 2 \sigma^{2}\right] /(2 \pi)^{1 / 2} \sigma D_{0}^{2}$

which is non-Gaussian even if $\sigma=\sigma_{\varpi_{0}}$ corresponds to a Gaussian distribution. The mathematical expectation is

$\left\langle D_{0}\right\rangle=\frac{1}{(2 \pi)^{1 / 2} \sigma} \int_{-\infty}^{+\infty} \frac{\exp \left[-\left(\varpi_{0}-\varpi\right)^{2} / 2 \sigma^{2}\right]}{\varpi_{0}} \mathrm{~d} \varpi_{0}$

and Smith \& Eichhorn (1996) calculated the ratio

$\Gamma=\left\langle D_{0}\right\rangle \varpi=\left\langle D_{0}\right\rangle / D$

and published a diagram of $\Gamma$ against the relative error

$\alpha=\sigma / \varpi$

(their Fig. 1 p. 213). For $\alpha \leq 0.74$ they obtain $\Gamma \geq 1$ (that is, the distance $\left\langle D_{0}\right\rangle$ is an overestimate) with a maximum of $\Gamma \simeq 1.28$ for $\alpha \simeq 0.49$. On the contrary, an underestimate $(\Gamma \leq 1)$ is observed for $\alpha \geq 0.74$, which increases for increasing $\alpha$. Of course $\Gamma \longrightarrow 1^{+}$for $\alpha \longrightarrow 0$.

The relation between pdfs

$g_{1}\left(\varpi / \varpi_{0}\right)=g_{1}\left(\varpi_{0} / \varpi\right)$ 
Table 2. The mean value of true parallaxes $\varpi$ for each photometric group. The theoretical correction factor $\Gamma$ leads to estimated means $\langle D\rangle_{\text {est }}$ close to the values of $1 /\langle\varpi\rangle$ (their ratio is $\rho$ in Col. 8). The quantities in Cols. 9 and 10 are on the contrary unusable. They are replaced by those in Cols. 11 and 12 (see text for details).

\begin{tabular}{|c|c|c|c|c|c|c|c|c|c|c|c|}
\hline $\mathrm{G}$ & $n$ & $\langle\varpi\rangle \pm \sigma_{\varpi}$ & $\Gamma$ & $\left\langle\frac{1}{\varpi}\right\rangle$ & $\langle D\rangle_{\text {est }}$ & $\frac{1}{\langle\varpi\rangle}$ & $\rho$ & $\left\langle\frac{\varpi}{|\sin b|}\right\rangle \pm \sigma$ & $\frac{1}{\left\langle\frac{\omega}{\sin b \mid}\right\rangle}$ & $\langle|z|\rangle$ & $\langle|z|\rangle_{\mathrm{c}}$ \\
\hline & n.d. & mas & n.d. & $\mathrm{kpc}$ & $\mathrm{kpc}$ & $\mathrm{kpc}$ & n.d. & mas & $\mathrm{kpc}$ & $\mathrm{kpc}$ & $\mathrm{kpc}$ \\
\hline $\mathrm{HCO}$ & 4 & $0.95 \pm 0.22$ & 1.07: & 1.11: & 1.04: & 1.06 & 0.98: & $5.0 \pm 0.7:$ & $0.201:$ & 0.20 : & 0.19: \\
\hline $\mathrm{HC} 1$ & 25 & $1.09 \pm 0.52$ & 1.28 & 1.07 & 0.84 & 0.92 & 0.91 & $2.7 \pm 1.9$ & 0.377 & 0.55 & 0.43 \\
\hline $\mathrm{HC} 2$ & 27 & $1.04 \pm 0.60$ & 1.23 & 1.19 & 0.97 & 0.96 & 1.01 & $13 \pm 51$ & 0.077 & 0.71 & 0.58 \\
\hline HC3 & 17 & $0.68 \pm 0.26$ & 1.24 & 1.61 & 1.30 & 1.48 & 0.88 & $270 \pm 1100$ & 0.004 & 0.62 & 0.50 \\
\hline $\mathrm{HC} 4$ & 13 & $0.80 \pm 0.29$ & 1.22 & 1.41 & 1.16 & 1.26 & 0.92 & $3.4 \pm 3.4$ & 0.292 & 0.67 & 0.55 \\
\hline HC5 & 18 & $0.85 \pm 0.19$ & 1.06 & 1.24 & 1.17 & 1.17 & 1.02 & $4.2 \pm 3.8$ & 0.240 & 0.48 & 0.46 \\
\hline CV1 & 31 & $1.21 \pm 0.67$ & 1.25 & 1.06 & 0.85 & 0.83 & 1.02 & $66 \pm 210$ & 0.015 & 0.18 & 0.15 \\
\hline $\mathrm{CV} 2$ & 43 & $1.33 \pm 0.59$ & 1.28 & 0.91 & 0.71 & 0.75 & 0.95 & $21 \pm 44$ & 0.048 & 0.17 & 0.16 \\
\hline CV3 & 32 & $1.18 \pm 0.93:$ & 0.97: & 1.11 & 1.14: & 0.84 & 1.36: & $12 \pm 15$ & 0.085 & 0.20 & 0.20 \\
\hline CV $3 *$ & 31 & $1.03 \pm 0.44$ & 1.27 & 1.14 & 0.90 & 0.97 & 0.93 & $13 \pm 15$ & 0.078 & 0.20 & 0.16 \\
\hline CV4 & 28 & $1.27 \pm 0.61$ & 1.28 & 0.97 & 0.76 & 0.79 & 0.96 & $300 \pm 1500$ & 0.003 & 0.17 & 0.13 \\
\hline CV5 & 28 & $1.46 \pm 0.68$ & 1.29 & 0.81 & 0.63 & 0.68 & 0.93 & $37 \pm 150$ & 0.027 & 0.18 & 0.14 \\
\hline CV6 & 25 & $1.45 \pm 0.69$ & 1.28 & 0.88 & 0.69 & 0.69 & 1.00 & $120 \pm 56$ & 0.008 & 0.23 & 0.18 \\
\hline CV7 & 6 & $0.96: \pm 0.24$ & 1.08: & 1.09: & 1.01 & 1.04: & 0.97: & $7.8 \pm 7.1:$ & $0.128:$ & 0.33: & 0.30 : \\
\hline SCV & 3 & $0.96: \pm 0.30$ & 1.15: & 1.11: & 0.97 & 1.05: & 0.92: & $9.0 \pm 5.4:$ & $0.111:$ & $0.14:$ & 0.13: \\
\hline
\end{tabular}

is verified only for a distribution uniform in true parallax, an unrealistic hypothesis. It was shown instead that $N(\varpi) \propto \varpi^{-2.35}$ (Paper I and Sect. 2), and thus

$g_{2.35}\left(\varpi / \varpi_{0}\right) \neq g_{1}\left(\varpi_{0} / \varpi\right)$.

The calculations of Smith \& Eichhorn should not apply, in principle, to the present case. The estimate of the mean distance obtained for every photometric group from

$\langle D\rangle_{\mathrm{est}}=\langle 1 / \varpi\rangle / \Gamma$

is however close to the value of $1 /\langle\varpi\rangle$ (see Table 2 ). We introduce the ratio

$\rho=\langle D\rangle_{\mathrm{est}} /(1 /\langle\varpi\rangle)=\langle\varpi\rangle\langle D\rangle_{\mathrm{est}}$.

The mean of the 15 values quoted in Table 2 is

$\langle\rho\rangle \simeq 0.98 \pm 0.11$

Removing the exceptionally high value $\varpi \simeq 5.68$ mas deduced for C2803 = U Hya, since it is located at $4.8 \sigma_{\varpi}$ of $\langle\varpi\rangle \simeq 1.18$, the $\mathrm{CV}^{*}$ sample was obtained. Ignoring the CV3 sample $(n=14)$ leads to

$\rho \simeq 0.96 \pm 0.04$

and, alternatively, excluding $\mathrm{CV}^{*}(n=14)$,

$\rho \simeq 0.99 \pm 0.12$.

The conclusion $\rho \simeq 1$ to within the errors may thus be accepted. The $\Gamma$-factor of Smith \& Eichhorn (1996) still applies here. The distributions of true parallaxes actually derived in Sect. 5 of Paper 1, do mimic the Gaussian shape.

\subsection{Mean distances $\langle|z|\rangle$ to the Galactic plane}

Considering the distances from the Galactic plane

$|z|=|\sin b| / \varpi$

where $b$ is the galactic latitude, the average

$\langle 1 /|z|\rangle=\langle\varpi /|\sin b|\rangle$

is calculated on a homogeneous linear function of $\varpi$ and thus suffers from no (Smith-Eichhorn) bias. It is however strongly influenced by the stars close to the Plane $(b \rightarrow 0 \Rightarrow \varpi /|\sin b| \rightarrow+\infty)$ : see Col. 9 in Table 2. The reciprocals of those quantities exhibit unrealistic values (Col. 10 of Table 2). We prefer to admit that the $\Gamma$-factor still applies to the biased values

$\langle|z|\rangle=\langle|\sin b| / \varpi\rangle$

leading to the corrected estimates

$\langle|z|\rangle_{\mathrm{c}}=\langle|z|\rangle / \Gamma$.

They are quoted respectively in Cols. 11 and 12 of Table 2.

We observe that the values for the HC-groups are much larger than those of the CV-groups. We find respectively

$\left[\langle|z|\rangle_{\mathrm{c}}\right]_{\mathrm{HC}} \simeq(0.50 \pm 0.06) \mathrm{kpc}$

for the HCi-groups ( $i=1$ to $5 ; 104$ stars), and for the CV-groups ( $j=1$ to $6 ; 187$ stars, CV3 adopted)

$\left[\langle|z|\rangle_{\mathrm{c}}\right]_{\mathrm{CV}} \simeq(0.16 \pm 0.03) \mathrm{kpc}$.

If $\mathrm{CV} 3$ is replaced by $\mathrm{CV} 3^{*}$, the result is

$\left[\langle|z|\rangle_{\mathrm{c}}\right]_{\mathrm{CV}} \simeq(0.15 \pm 0.02) \mathrm{kpc}$. 
The ratio is thus

$\left[\langle|z|\rangle_{\mathrm{c}}\right]_{\mathrm{HC}} /\left[\langle|z|\rangle_{\mathrm{c}}\right]_{\mathrm{CV}} \simeq 3.13$ or 3.33

respectively, adopting Eq. (28) or Eq. (29).

The CV-stars are, on average, about three times more concentrated towards the Plane than the HC-ones. A clear-cut jump between both regimes appears at HC5-CV1 two groups which are however close to each other in terms of mean effective temperatures (Bergeat et al. 2001), and also on the grounds of mean absolute magnitudes (Col. 2 of Table 3 and Knapik et al. 2001). We emphasize that those results were quite unexpected when the definitions of our photometric groups were adopted, precisely on the basis of photometric criteria only. In Table 2, the values quoted for $\mathrm{HC} 0, \mathrm{SCV}$ and CV7 are only indicative.

\section{Absolute magnitudes of the photometric groups}

In Sect. 7.4 of Paper I, we concluded that the subset of the bright $\mathrm{N}$-variables (CV-stars) in the HIPPARCOS sample is little affected by the Malmquist bias, while the opposite situation prevails for the faint hotter R-type stars (HC-stars). This statement applies to the evaluation of mean absolute magnitudes for the $\mathrm{HC}$ and $\mathrm{CV}$-groups, and thus to mean luminosities. Despite the differences between those two categories, only a Gaussian curve could successfully be fitted to the distribution of the visual absolute magnitudes, namely Eq. (5). Along the sequence of the thirteen $\mathrm{HC}$ and $\mathrm{CV}$-groups (HC0 to HC5 and then CV1 to CV7) which is also an effective temperature sequence (Bergeat et al. 2001), the mean absolute visual magnitude hardly changes, contrary to its bolometric counterpart. The latter displays a much wider range (Table 3 with results from Knapik et al. 2001). Mean bolometric corrections were computed (Col. 5 of Table 3 ), and then mean absolute visual magnitudes (Col. 6) from

$\left\langle M_{V}\right\rangle=\left\langle M_{\mathrm{bol}}\right\rangle-\left\langle B C_{V}\right\rangle \cdot$

A wide interval of nearly uniform $\left\langle M_{V}\right\rangle$ is observed which ranges approximately from $\mathrm{HC} 4$ to $\mathrm{CV} 6$ (8 groups) with

$\left\langle\left\langle M_{V}\right\rangle\right\rangle=(-1.74 \pm 0.11) \pm 0.04$.

It is flanked by two increasing (in $\left\langle M_{V}\right\rangle$ ) branches, respectively $\mathrm{HC} 1$ to $\mathrm{HC} 4$ and $\mathrm{CV} 6-\mathrm{CV} 7$. Finally, this is roughly consistent with the only Gaussian (5). The diagrams $M_{V}(5 \log \varpi)$ and $M_{\mathrm{bol}}(5 \log \varpi)$ show that the influence of the Malmquist bias is maximum in the $\mathrm{HC1}$ $\mathrm{HC} 2$ groups. In the absence of bias, the stars should populate a complete horizontal rectangular strip (no dependence). The straight lines shown in Figs. 2 and 3 correspond to the limiting magnitudes in $M_{V}$ as given by Eqs. (3) and (4) substituted in Eq. (2), for the HC2 and CV4-groups respectively. It can be seen that they delineate two left-inferior triangular portions which are almost empty. The triangular area for CV4 in Fig. 3 is much smaller than its counterpart for $\mathrm{HC} 2$ in Fig. 2.
Table 3. Mean values and ranges of the absolute bolometric magnitudes, mean bolometric corrections for the $V$-bandpass, and mean absolute visual magnitudes, for each photometric group: all quantities being adapted from Knapik et al. (2001); (a) without $\mathrm{C} 4247$ which is brighter than the three other stars; (b) without $\mathrm{C} 2334$ which is underluminous; (c) values corrected from the influence of the Malmquist bias.

\begin{tabular}{cccccc}
\hline $\mathrm{G}$ & $\left\langle M_{\mathrm{bol}}\right\rangle$ & $\left\langle M_{\mathrm{bol}}\right\rangle^{\mathrm{c}}$ & (inf; sup) & $\left\langle\mathrm{BC}_{V}\right\rangle$ & $\left\langle M_{V}\right\rangle$ \\
\hline $\mathrm{HC} 0$ & -1.71 & & $-0.92 ;-2.98$ & -0.13 & -1.58 \\
$\mathrm{HC}^{(a)}$ & -1.32 & & $-0.92 ;-1.81$ & -0.13 & -1.19 \\
$\mathrm{HC} 1$ & -1.06 & & $-0.35 ;-2.11$ & -0.60 & -0.46 \\
$\mathrm{HC}^{(c)}$ & & -0.74 & $-0.00 ;-1.80$ & -0.60 & -0.14 \\
$\mathrm{HC} 2$ & -1.22 & & $-0.58 ;-2.15$ & -0.60 & -0.62 \\
$\mathrm{HC} 2^{(c)}$ & & -1.02 & $-0.30 ;-2.00$ & -0.60 & -0.42 \\
$\mathrm{HC} 3$ & -1.99 & & $-1.15 ;-3.36$ & -0.88 & -1.11 \\
$\mathrm{HC} 4$ & -3.00 & & $-2.11 ;-4.54$ & -1.16 & -1.84 \\
$\mathrm{HC} 5$ & -3.37 & & $-2.81 ;-4.12$ & -1.70 & -1.67 \\
$\mathrm{CV} 1$ & -3.72 & -3.62 & $-3.01 ;-4.79$ & -2.07 & -1.65 \\
$\mathrm{CV} 2$ & -4.34 & -4.24 & $-3.65 ;-5.35$ & -2.42 & -1.92 \\
$\mathrm{CV} 3$ & -4.39 & -4.29 & $-3.74 ;-5.32$ & -2.64 & -1.75 \\
$\mathrm{CV} 4$ & -4.66 & -4.56 & $-3.91 ;-5.80$ & -2.83 & -1.83 \\
$\mathrm{CV} 5$ & -4.82 & -4.72 & $-4.24 ;-5.60$ & -3.17 & -1.65 \\
$\mathrm{CV} 6$ & -5.05 & -4.95 & $-4.49 ;-5.79$ & -3.43 & -1.62 \\
$\mathrm{CV} 7$ & -5.43 & & $-4.60 ;-6.80$ & -4.62 & -0.81 \\
$\mathrm{CV} 7^{(b)}$ & -5.81 & & $-5.20 ;-6.70$ & -4.62 & -1.19 \\
$\mathrm{SCV}$ & -5.77 & & $-5.51 ;-6.06$ & & \\
\hline
\end{tabular}

Adopting the $\left\langle B C_{V}\right\rangle$ means from Table 3 we may use in the $M_{\mathrm{bol}}$-diagrams, the straight lines

$\left(M_{\mathrm{bol}}\right)_{1}=\left(M_{V}\right)_{1}+\left\langle B C_{V}\right\rangle$

where $\left(M_{V}\right)_{1}$ is expressed as mentioned above. Returning to Figs. 2 and 3, the influence of the Malmquist bias can be estimated by another method, (1) re-constructing the complete horizontal strip from a Monte-Carlo simulation, (2) restricting the averages to the complete portions, e.g. $\varpi \geq 0.83$ mas for HC2 and $\varpi \geq 0.67$ mas for CV4. Finally, we have derived corrections according to

$\left\langle M_{\mathrm{bol}}\right\rangle^{\prime}=\left\langle M_{\mathrm{bol}}\right\rangle+\delta\left\langle M_{\mathrm{bol}}\right\rangle \cdot$

We found $\delta\left\langle M_{\mathrm{bol}}\right\rangle \simeq 0.32$ for HC1 and 0.20 for HC2 which are by far the largest corrections met. For CV1 to CV6, we obtained smaller clustered values and we adopt there

$\delta\left\langle M_{\mathrm{bol}}\right\rangle=0.10 \pm 0.03$

a correction which will be applied since it is reasonably well-established. The values for $\mathrm{HC} 3, \mathrm{HC} 4$ and $\mathrm{HC} 5$ are poorly determined, and we could settle for $\delta\left\langle M_{\mathrm{bol}}\right\rangle \leq$ 0.10 . No correction will be applied in this latter case, just like for the $\mathrm{HC} 0, \mathrm{CV} 7$ and SCV-groups.

We conclude that the corrections on mean absolute magnitudes due to the Malmquist bias are small (and could have been neglected) when compared to the large 


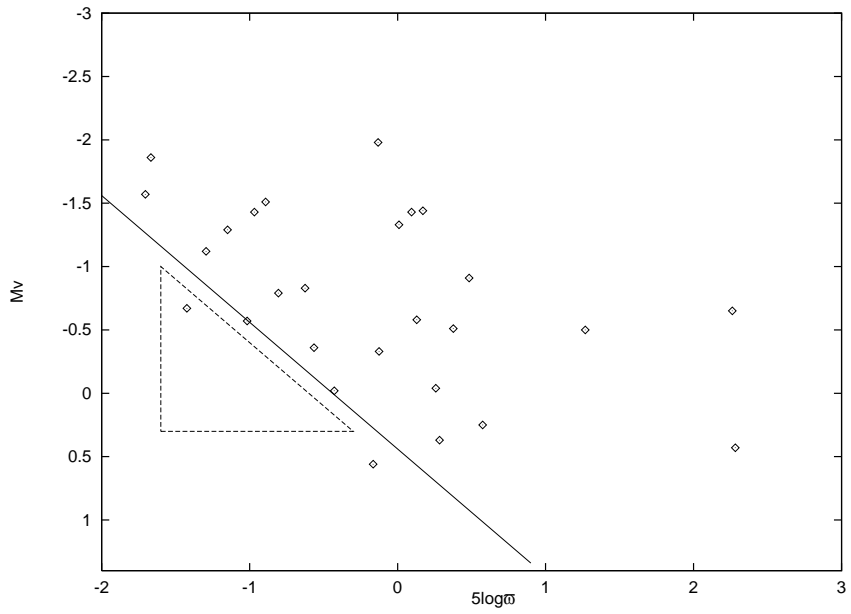

Fig. 2. The influence of the Malmquist bias on the HIPPARCOS sample of HC2-stars.

ranges (inf; sup) in Table 3 . The intrinsic dispersions of the absolute bolometric magnitudes in a given group are enlarged by substantial errors on parallaxes.

\section{Space distributions and densities}

\subsection{Generalities}

We plotted diagrams of $z=\sin b / \varpi$ vs. $r=\cos b / \varpi$ for every group, and then, globally, for the HC- and CVstars respectively. Having found no marked difference between both Galactic hemispheres, we finally used $(r,|z|)$ coordinates in diagrams. Rectangular domains of widths such as $0 \leq r \leq 1.6$ to $1.8 \mathrm{kpc}$, are populated. Their heights are $|z| \leq 1.8 \mathrm{kpc}$ for the HC-stars and $|z| \leq 0.5$ to $0.6 \mathrm{kpc}$ for the CV-stars (a ratio of about 3: Eq. (30)).

The influence of the Malmquist bias was roughly compensated at by applying Eq. (6) with

$\varpi=\left(r^{2}+|z|^{2}\right)^{-1 / 2}$.

The term $M_{V}+1.5$ in Eq. (5) was replaced by $M_{V}+1.21$ for HC-stars and $M_{V}+1.73$ for CV-stars, as obtained from data in Table 3. The new Eq. (7) for HC-stars is thus

$\left(x_{1}\right)_{\mathrm{HC}} \simeq\left(M_{V 1}+1.21\right) / 1.06 \simeq 1.557+4.708 \log \varpi$

where $\left(V_{l}^{\prime}\right)_{\mathrm{HC}} \simeq 10.44$ from Eq. (3), while

$\left(x_{1}\right)_{\mathrm{CV}} \simeq\left(M_{V 1}+1.73\right) / 1.06 \simeq 1.226+4.708 \log \varpi$

is obtained for $\mathrm{CV}$-stars from $\left(V_{1}^{\prime}\right)_{\mathrm{CV}} \simeq 9.57$ i.e. Eq. (4). The $f$-factors were applied to frequencies in square bins of sizes 0.16 and $0.32 \mathrm{kpc}$ for $\mathrm{CV}$ - and HC-stars, respectively. Cumulative counts were then performed as a function of $r$ in the same intervals, all contributions of $|z|$-ranges being added. Finally, surface densities projected on the Plane were derived. They are nearly uniform in cylinders of increasing radii, up to $r \leq 0.8 \mathrm{kpc}$ for $\mathrm{CV}$-stars, and $r \leq 1.28 \mathrm{kpc}$ for HC-stars. They amount to $40-50 \mathrm{kpc}^{-2}$ and $15-20 \mathrm{kpc}^{-2}$ respectively. For wider cylinders, we

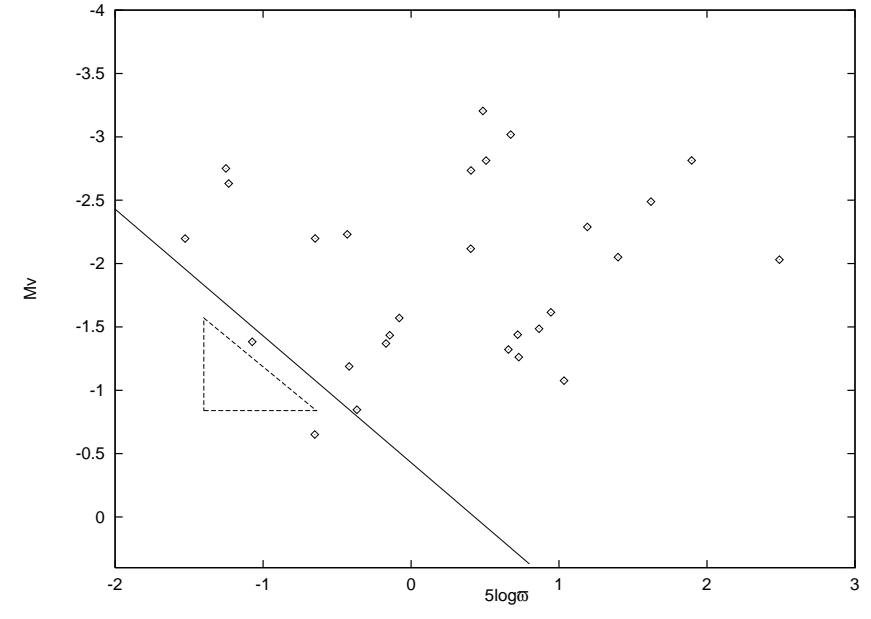

Fig. 3. The influence of the Malmquist bias on the HIPPARCOS sample of CV4-stars.

found slowly decreasing values, possibly due to underestimated Malmquist corrections: e.g. zero frequencies in remote bins remained so after the multiplicative correction is applied. Since no concentration of carbon stars is expected in the neighborhood of the Sun we restrict any further analysis to the above-mentioned cylinders.

\subsection{Distributions vs. $|z|$}

We denote by $N$ the space density of stars in $\mathrm{kpc}^{-3}$, corrected for the Malmquist bias (Sect. 5.1). An exponential decrease of the space density with $|z|$ is usually derived for various stars (Gilmore \& Reid 1983; Freeman 1987a, 1987b; Gould et al. 1996). Hence we studied diagrams (not shown here) of $\ln N(|z|)$ vs. $|z|$, according to

$N(|z|)=N_{0} \exp \left(-|z| / z_{0}\right)$

where $z_{0}$ is the distance scale. Linear fits were obtained from the least square method, yielding the slope $-1 / z_{0}$ and the intercept $\ln N_{0}$, with the corresponding uncertainties and the coefficient of determination (Table 4).

The high accuracy of the $\mathrm{CV}$ fit is due to the first three points at $0.08,0.24$ and $0.4 \mathrm{kpc}$, which have very high weights ( $95.5 \%$ of the total). It is probably accidental. Increasing the radius of the sampled cylinder to $0.96 \mathrm{kpc}$ leads to the same slope within $1 \%$ and to a reduction of $17 \%$ of $N_{0}$. For the HC-sample, which is about half the $\mathrm{CV}$-one in star number, the accuracy is lower. Varying the cylinder radius from $1.28 \mathrm{kpc}$ to $1.6 \mathrm{kpc}$ increases the slope from $1.05 \pm 0.07$ to $1.18 \pm 0.08$ with $\ln N_{0} \simeq 2.88$. Unacceptable scatter is obtained when adopting $0.96 \mathrm{kpc}$ (Poisson error bars). Finally, the adopted solutions are those given in Table 4, i.e. for $0.8 \mathrm{kpc}$ (CV-sample) and $1.28 \mathrm{kpc}$ (HC-sample) respectively. Both coefficients of determination are very high. The projected densities (on the Plane) $z_{0} N_{0}$, and space densities in the Plane $N_{0} / 2$ are also quoted for both samples. The ratio ( $\mathrm{CV}$ to $\mathrm{HC}$ ) of the latter is about 12.6 while that of the former is only 2.5 . 
Table 4. The parameters of the modeled space distributions of the HC- and CV-stars according to Eq. (39), as obtained in adopted cylinders radii of Col. 3. The corresponding space densities in the Plane, vertical distance scales, and projected space densities, are given in Cols. 7 to 9. Values corrected for missing CV6-CV7 and SCV-stars, are quoted in Cols. 11 and 12.

\begin{tabular}{|c|c|c|c|c|c|c|c|c|c|c|c|c|c|}
\hline Groups & $n$ & $\begin{array}{l}r_{\mathrm{c}} \\
\mathrm{kpc}\end{array}$ & $\ln N_{0}$ & $\begin{array}{c}1 / z_{0} \\
\mathrm{kpc}^{-1}\end{array}$ & $\rho^{2}$ & $\begin{array}{l}N_{0} / 2 \\
\mathrm{kpc}^{-3}\end{array}$ & $\begin{array}{l}z_{0} \\
\mathrm{kpc}\end{array}$ & $\begin{array}{l}z_{0} N_{0} \\
\mathrm{kpc}^{-2}\end{array}$ & $\mathrm{NF}$ & $\begin{array}{l}N_{0}^{\prime} / 2 \\
\mathrm{kpc}^{-3}\end{array}$ & $\begin{array}{c}\left(z_{0} N_{0}\right)^{\prime} \\
\mathrm{kpc}^{-2}\end{array}$ & NF' & Population \\
\hline $\mathrm{HC}$ & 104 & 1.28 & $\begin{array}{c}3.065 \\
\pm 0.094\end{array}$ & $\begin{array}{c}.05 \\
\pm 0.07\end{array}$ & 1.00 & $\begin{array}{l}10.6 \\
\pm 1.0\end{array}$ & $\begin{array}{c}0.95 \\
\pm 0.06\end{array}$ & $\begin{array}{c}20.4 \\
\pm 3.0\end{array}$ & 0.073 & & & 0.067 & Thick disk + Halo \\
\hline $\mathrm{HC}^{\prime}$ & 81 & & $\begin{array}{c}2.81 \\
\pm 0.09\end{array}$ & & & $\begin{array}{c}8.3 \\
\pm 0.8\end{array}$ & & $\begin{array}{c}15.9 \\
\pm 2.3\end{array}$ & 0.057 & & & 0.052 & Thick disk \\
\hline $\begin{array}{r}\mathrm{CV} \\
+\mathrm{SCV}\end{array}$ & 195 & 0.8 & $\begin{array}{c}5.592 \\
\pm 0.021\end{array}$ & $\begin{array}{c}5.33 \\
\pm 0.09\end{array}$ & 0.95 & $\begin{array}{c}134 \\
\pm 3\end{array}$ & $\begin{array}{c}0.188 \\
\pm 0.003:\end{array}$ & $\begin{array}{c}50.3 \\
\pm 2.0\end{array}$ & 0.927 & $\begin{array}{c}147 \\
\pm 3.3\end{array}$ & $\begin{array}{c}55.3 \\
\pm 2.2\end{array}$ & 0.933 & Thin disk \\
\hline
\end{tabular}

This is a direct consequence of the distance scales $z_{0}$ differing from one another, with a ratio of nearly 0.2 .

The vertical distance scale $\left(z_{0}\right)_{\mathrm{HC}} \simeq 0.95 \mathrm{kpc}$ falls in the middle of the $0.6-1.45 \mathrm{kpc}$ range of values published for the thick disk. It is in excellent agreement with recently published values such as $0.86 \pm 0.20 \mathrm{kpc}$ by Ojha (2001) or $0.8 \leq z_{0} \leq 1.2 \mathrm{kpc}$ by Kerber et al. (2001). This disk represents a structure much thicker than the classical thin disk to which our $\left(z_{0}\right)_{\mathrm{CV}} \simeq 0.19 \mathrm{kpc}$ corresponds well. The latter value is close to that of Kerschbaum \& Hron (1992) for C-rich SRbs and Miras, viz. $0.18 \mathrm{kpc}$. Claussen et al. (1987) and Groenewegen et al. (1992) found $0.2 \mathrm{kpc}$ for various samples of carbon giants. Van Eck \& Jorissen (2000) derived $0.22 \mathrm{kpc}$ for (bright) intrinsic-S stars and $0.58 \mathrm{kpc}$ for (faint) extrinsic-S stars which are brighter $\left(\left\langle M_{\mathrm{bol}}\right\rangle \simeq-3.1\right)$ than the HC-stars $\left(\left\langle M_{\mathrm{bol}}\right\rangle \simeq-1.8\right)$.

The population of the thick disk was also named "Intermediate Population II" (IPII; e.g. Majewski 1993 for a review). Not all spiral galaxies exhibit such a structure and its (dynamical) origin is unknown: various theories like violent thin disk heating by satellite accretion (model 6 in Table 1 of Majewski's paper) were proposed. Here we take advantage of the natural separation of carbon giants on the grounds of their SEDs, into two categories (HC and $\mathrm{CV}$ ), whose space distributions differ markedly (i.e. thick disk and thin disk respectively). Of course, mutual contamination of both samples is likely at HC5-CV1, and halo CH stars are also involved. There is no such separation for faint oxygen-rich stars and the studies of the thick disk are difficult. Gilmore \& Reid (1983) modeled the distribution of the faintest M-dwarfs with two exponentials of distance scales $0.3 \mathrm{kpc}$ and $1.45 \mathrm{kpc}$, and a normalization factor (NF) of $2 \%$ for the thick disk population. Making use of HST data, Gould et al. (1996) found $0.323 \mathrm{kpc}$ and $0.656 \mathrm{kpc}$, and $20 \%$. The numbers for carbon giants (Table 4) are $0.19 \mathrm{kpc}$ and $0.95 \mathrm{kpc}$, and a NF of $7.3 \%$ for the thick disk + halo $(5.7 \%$ for thick disk).

We conclude that the distribution of the HC-stars is typical of a thick disc component contaminated by a true spheroidal contribution, to which the 23 hot HC-stars classified as $\mathrm{CH}$ stars belong. The CV-stars are found as a component of the (old) thin disk. Since the CV6-CV7 objects with thick circumstellar dust shells and the SCVstars are almost absent from the HIPPARCOS sample, we propose a tentative $10 \%$ correction to the $\mathrm{CV}$-densities in Cols. 11-12 of Table 4 . Then the normalization factors
(NF') should be $6.7 \%$ and $93.3 \%$ for the thick disk + halo and thin disk respectively ( $5.2 \%$ for thick disk).

\section{Space densities in terms of photometric groups}

There were 567 carbon stars (597 SEDs) successfully studied in our previous papers, including

$$
\begin{aligned}
& \text { - } 130 \text { HC-stars (131 SEDs) i.e. 22.9\%, } \\
& \text { - } 423 \text { CV-stars (449 SEDs) i.e. } 74.6 \% \text {, } \\
& \text { - } 14 \text { SCV-stars (17 SEDs) i.e. 2.5\%, }
\end{aligned}
$$

to which should be added 22 stars (24 SEDs) classified in oxygen-rich groups. We ignore here nearly $100 \mathrm{BaII}$ stars classified in oxygen-rich groups that were treated separately (Bergeat \& Knapik 1997). We emphasize that 101 carbon stars amongst the above-mentioned 567 stars (i.e. $17.8 \%$ ) are not affected by any appreciable interstellar extinction $\left(E_{B-V} \leq 0.02\right)$. The distribution of those 567 carbon stars in our 14 groups is shown in Fig. 4 . It is obviously asymmetric and bimodal (HC and CV-zones).

The corresponding projected densities in the Plane are given in Table 5 (calibration from Table 4). The value for the CV7-group is certainly underestimated by a factor of typically two. The average frequency is about 40.5 stars per group, but the distribution is quite non-uniform. Two peaks are observed at nearly $\mathrm{HC} 1-\mathrm{HC} 2$ and CV5-CV6 respectively, with a minimum at $\mathrm{HC} 3-\mathrm{HC} 4$. This is consistent with the mean true parallaxes quoted in Col. 3 of Table 2: they show a minimum in the same range. Those rarer HC3 to HC5 stars are observed farther from Sun on average. We also notice a minimum at CV4. It can be considered an artifact of our classification which is tighter in effective temperatures at CV4-CV5 than it is at CV3CV4 or CV5-CV6 (Bergeat et al. 2001). If only one group (CV4-5) was adopted instead, it would have been the most populated one in the whole diagram. This interpretation will be confirmed in Sect. 7 .

The present statistics relies on a sample about twice the HIPPARCOS one. The existence is confirmed of two great families in those samples ( $\mathrm{HC}$ and $\mathrm{CV}+\mathrm{SCV}$ ), made of distinct stellar populations (Sect. 5).

\section{Space densities vs. effective temperatures}

The sequence of the HC- and CV-groups correlates well with that of decreasing effective temperatures from 
Table 5. The projected densities in $\mathrm{kpc}^{-2}$, of the $567 \mathrm{HC}-, \mathrm{CV}$ - and SCV-stars corresponding to data in Fig. 4 calibrated from densities $55.3 \pm 2.2$ for CV + SCV stars, and $20.4 \pm 3.0$ for HC-stars (Table 4); ${ }^{\text {a }}$ underestimated.

\begin{tabular}{cccccccccccccc}
\hline HC0 & HC1 & HC2 & HC3 & HC4 & HC5 & CV1 & CV2 & CV3 & CV4 & CV5 & CV6 & CV7 & SCV \\
\hline 0.6 & 4.9 & 4.6 & 3.3 & 3.2 & 3.8 & 5.8 & 7.7 & 8.2 & 5.7 & 10.1 & 12.6 & $3.6:^{\text {a }}$ & 1.6 \\
\pm 0.1 & \pm 0.7 & \pm 0.7 & \pm 0.5 & \pm 0.5 & \pm 0.6 & \pm 0.2 & \pm 0.3 & \pm 0.3 & \pm 0.2 & \pm 0.4 & \pm 0.5 & $\pm 0.1:$ & \pm 0.07 \\
\hline
\end{tabular}

Table 6. The projected densities in $\mathrm{kpc}^{-2}$, of the 432 carbon stars and related objects according to effective temperatures, and calibrated with a total density of $75.7 \pm 3.7$. The considered intervals are $\left(T_{\text {eff I }}, T_{\text {eff S }}\right) ;{ }^{a}$ underestimated.

\begin{tabular}{c|ccccccccccc}
\hline$T_{\text {eff S }}$ & 3.84 & 3.81 & 3.78 & 3.75 & 3.72 & 3.69 & 3.66 & 3.63 & 3.60 & 3.57 & 3.54 \\
$T_{\text {eff I }}$ & 3.81 & 3.78 & 3.75 & 3.72 & 3.69 & 3.66 & 3.63 & 3.60 & 3.57 & 3.54 & 3.51 \\
\hline PD & 0.70 & 0.35 & 1.40 & 0.53 & 2.28 & 3.33 & 3.15 & 4.90 & 3.15 & 3.15 & 6.48 \\
Err. & \pm 0.03 & \pm 0.02 & \pm 0.07 & \pm 0.03 & \pm 0.11 & \pm 0.16 & \pm 0.15 & \pm 0.24 & \pm 0.15 & \pm 0.15 & \pm 0.32 \\
\hline \hline$T_{\text {eff S }}$ & 3.51 & 3.48 & 3.45 & 3.42 & 3.39 & 3.36 & 3.33 & 3.30 & 3.27 & 3.24 \\
$T_{\text {eff I }}$ & 3.48 & 3.45 & 3.42 & 3.39 & 3.36 & 3.33 & 3.30 & 3.27 & 3.24 & 3.21 \\
\hline PD & 5.96 & 13.7 & 11.4 & 5.78 & 4.38 & $0.70:^{\mathrm{a}}$ & $1.93:^{\mathrm{a}}$ & $1.40:^{\mathrm{a}}$ & $0.70:^{\mathrm{a}}$ & $0.35:^{\mathrm{a}}$ \\
Err. & \pm 0.29 & \pm 0.67 & \pm 0.56 & \pm 0.28 & \pm 0.22 & $\pm 0.03:$ & $\pm 0.095:$ & $\pm 0.07:$ & $\pm 0.03:$ & $\pm 0.02:$ \\
\hline
\end{tabular}

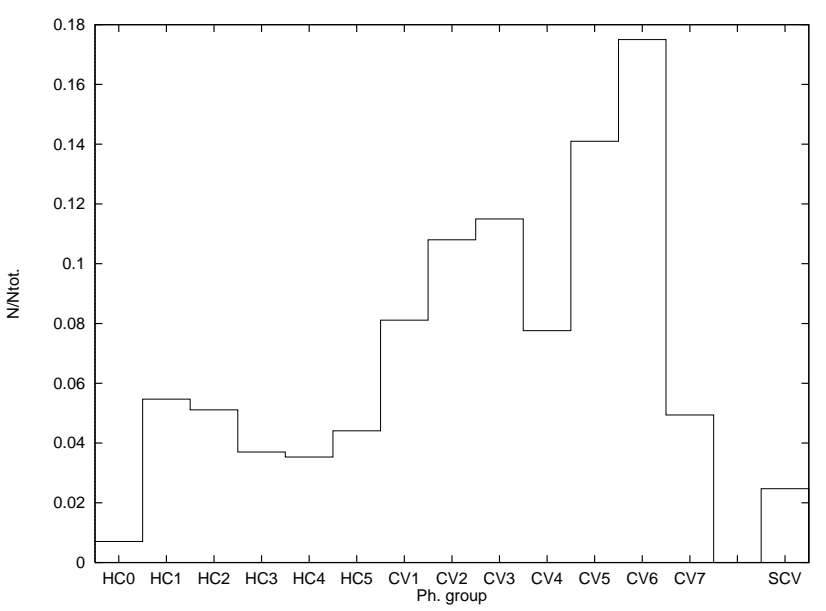

Fig. 4. The distribution of the 567 studied carbon stars in the fourteen groups. The scale is normalized for a total of unity.

Bergeat et al. (2001) for 432 SEDs. The values are distributed in the range

$$
3.21 \leq \log T_{\text {eff }} \leq 3.83 \quad \text { or } \quad 1620 \mathrm{~K} \leq T_{\text {eff }} \leq 6760 \mathrm{~K}
$$

Their distribution is shown in Fig. 5, the above range being subdivided into 21 intervals of 0.03 -width in $\log T_{\text {eff }}$. Projected densities in the Plane are given in Table 6 (calibration from Table 4). The results for $\log T_{\text {eff }}<3.36$ are probably underestimated due to missing CV7-objects.

The maximum of Fig. 5 is located near $\log T_{\text {eff }} \simeq$ 3.43-3.47 centered at $2820 \mathrm{~K}$, i.e. at nearly CV4 $\left(\left\langle T_{\text {eff }}\right\rangle \simeq 2775 \mathrm{~K}\right)$. The minimum of Fig. 4 at CV4 has no counterpart here. It is thus an artifact of the classification which is tighter in effective temperatures at CV4CV5. A relative minimum is hardly noticed in the range $3.54 \leq \log T_{\text {eff }} \leq 3.60$ or $3470 \mathrm{~K} \leq T_{\text {eff }} \leq 3980 \mathrm{~K}$ that is near HC5-HC3, which corresponds to the minimum in Fig. 4. The asymmetric distribution is still compatible

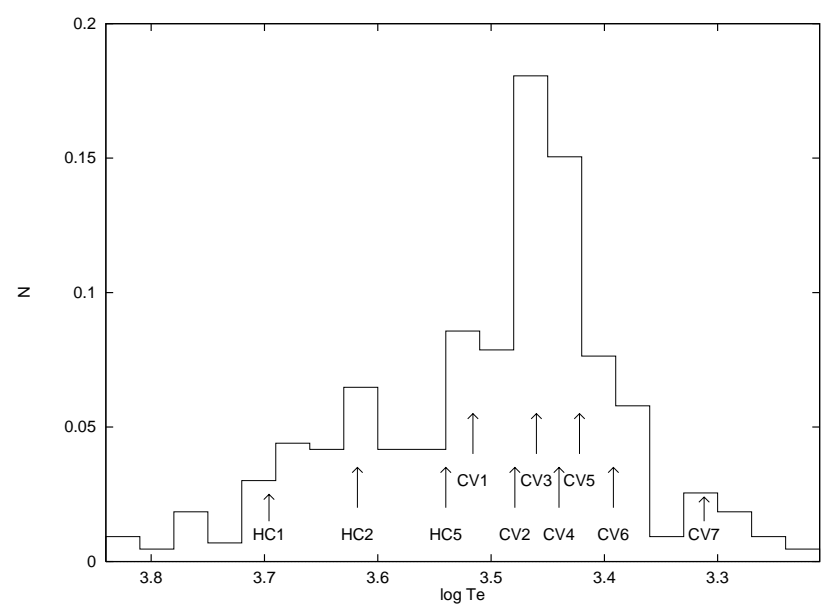

Fig. 5. The distribution of the 432 SEDs of carbon stars with effective temperatures (arrows point to mean values).

with the existence of the two families ( $\mathrm{HC}$ and $\mathrm{CV}$ ). An additional minimum is observed at $\log T_{\text {eff }} \simeq 3.33-3.36$ (2140-2290 K) since few CV6 or CV7 stars fall in this range. The calibration of effective temperatures is difficult here (Bergeat et al. 2001). Also part of the CV7stars are missing. The reality and meaning of this latter minimum is questionable. A systematic underestimate of nearly $100 \mathrm{~K}$ in the calibration of the CV7 stars cannot be ruled out. Alternatively, a true jump in $T_{\text {eff }}$ can intervene at the CV6-CV7 junction, as induced by substantial photospheric opacities from circumstellar dust at $T_{\text {eff }}<2400 \mathrm{~K}$.

\section{Mean radial and tangential velocities}

Prior to a detailed analysis of the velocities, we derive the mean values of the radial components $V_{\mathrm{r}}$, and those of the tangential ones from proper motions and parallaxes i.e.

$V_{\mathrm{t}} \simeq 4.738\left[\left(\mu_{\alpha} \cos \delta\right)^{2}+\mu_{\delta}^{2}\right]^{1 / 2} / \varpi \simeq 4.738 \mu / \varpi$. 
Table 7. A summary of the mean values for the five samples considered in Sect. 8: the whole samples for the CV and SCV-stars and for the HC-stars on one hand, and three subsamples of the latter on the other hand (see text for definitions).

\begin{tabular}{|c|c|c|c|c|c|c|c|c|c|c|}
\hline Type & $\mathrm{n}$ & $\begin{array}{l}\langle\varpi\rangle \\
\text { mas }\end{array}$ & $\begin{array}{l}\langle\mu\rangle \\
\text { mas }\end{array}$ & $\begin{array}{c}\left\langle\left|V_{\mathrm{r}}\right|\right\rangle \\
\mathrm{km} \mathrm{s}^{-1}\end{array}$ & $\begin{array}{r}\langle|z|\rangle \\
\mathrm{kpc}\end{array}$ & $\begin{array}{c}\langle|z|\rangle_{\mathrm{c}} \\
\mathrm{kpc}\end{array}$ & $\begin{array}{c}\left\langle\left|V_{\mathrm{t}}\right|\right\rangle \\
\mathrm{km} \mathrm{s}^{-1}\end{array}$ & $\begin{array}{c}\left\langle\left|V_{\mathrm{t}}\right|\right\rangle_{\mathrm{c}} \\
\mathrm{km} \mathrm{s}^{-1}\end{array}$ & $\begin{array}{c}\left\langle T_{\text {eff }}\right\rangle \\
\mathrm{K}\end{array}$ & $\left\langle M_{\mathrm{bol}}\right\rangle$ \\
\hline $\begin{array}{c}\mathrm{CV} \\
(+\mathrm{SCV})\end{array}$ & 195 & $1.28 \pm 0.68$ & $7.8 \pm 7.0$ & $23 \pm 19$ & $0.22 \pm 0.03$ & $0.19 \pm 0.03$ & $31 \pm 22$ & $27 \pm 19$ & 2720 & -4.6 \\
\hline $\mathrm{HC}$ & 104 & $0.93 \pm 0.46$ & $17.4 \pm 25$ & $47 \pm 57$ & $0.57 \pm 0.42$ & $0.50 \pm 0.37$ & $91 \pm 109$ & $80 \pm 95$ & 4350 & -1.8 \\
\hline $\begin{array}{l}\mathrm{HC} \\
(1)\end{array}$ & 81 & $0.96 \pm 0.50$ & $11.0 \pm 9.5$ & $44 \pm 45$ & $0.56 \pm 0.43$ & $0.49 \pm 0.38$ & $64 \pm 51$ & $56 \pm 45$ & 4300 & -1.7 \\
\hline $\begin{array}{l}\mathrm{HC} \\
(2)\end{array}$ & 12 & $1.05 \pm 0.59$ & $9.9 \pm 12.1$ & $35 \pm 22$ & $0.32 \pm 0.28$ & $0.28 \pm 0.25$ & $43 \pm 28$ & $37 \pm 24$ & $\begin{array}{c}5400 \\
\pm 1000\end{array}$ & -1.8 \\
\hline $\begin{array}{r}\mathrm{HC} \\
(3)\end{array}$ & 23 & $0.92 \pm 0.32$ & $51 \pm 65$ & $98 \pm 100$ & $0.63 \pm 0.38$ & $0.56 \pm 0.33$ & $197 \pm 187$ & $173 \pm 163$ & $\begin{array}{l}4170 \\
\pm 550\end{array}$ & -1.6 \\
\hline
\end{tabular}

The radial velocities were extracted from CDS at Strasbourg and catalogues and references there indexed. The two proper motion components were taken from the HIPPARCOS catalogue (ESA 1997) and the estimated true parallaxes from Paper I. The space velocities are

$V=\left(V_{\mathrm{t}}^{2}+V_{\mathrm{r}}^{2}\right)^{1 / 2}$.

The average values $\left\langle V_{\mathrm{t}}\right\rangle$ are however affected by the bias described by Smith \& Eichhorn (1996) in the same way as distances $D$ to the observer and distances $|z|$ to the Plane (Sect. 3 and Table 2). This is not the case of $\left\langle V_{\mathrm{r}}\right\rangle$.

It is remarkable that all confirmed $\mathrm{CH}$ stars on the one hand and the HdC stars on the other hand, are all contained in the HC photometric groups (specifically the "hot carbon stars" with $T_{\text {eff }} \geq 3400 \mathrm{~K}$, Bergeat et al. 2001). Conversely the ${ }^{13} \mathrm{C}$-rich stars or J-stars, are found in both the $\mathrm{HC}$ and $\mathrm{CV}$-groups. Mean values and dispersions are quoted in Table 7 for five samples

- the CV-stars (classified into groups CV1 to CV7);

- the HC-stars (classified into groups HC0 to HC5);

- (1) the HC-stars without CH (spectroscopy);

- (2) the HC-stars classified HdC and/or RCB-variables;

- and (3) the HC-stars classified as $\mathrm{CH}$ stars.

The reader should remember that radial velocities may be affected by atmospheric velocities, especially in Miras, and by orbital velocities in binary systems. Both categories of objects are however few in the present samples, and statistical cancellation of random contributions in either direction is expected. We attempt a rough correction of this bias making use of Eq. (26) and of

$\left\langle V_{\mathrm{t}}\right\rangle_{\mathrm{c}}=\left\langle V_{\mathrm{t}}\right\rangle /\langle\Gamma\rangle$

where $\langle\Gamma\rangle \simeq 1.14$ is the estimated mean for our range of accuracies, of the ratio $\Gamma$ defined in Eq. (14). Both uncorrected and corrected values are quoted in Table 7 .

Concerning velocities, marked differences are observed. The CV-stars display the smallest mean proper motions and velocities (with $\left\langle V_{\mathrm{t}}\right\rangle_{\mathrm{c}} \simeq\left\langle\left|V_{\mathrm{r}}\right|\right\rangle$ ) and consistently, a mean distance to the Galactic plane less than those of the HC-stars. For the HC-stars, the mean proper motions and radial velocities are $2-3$ times the respective values for the
CV-stars. The velocities of the HC(1)-sample (CH stars omitted) are twice those of the CV-sample. Finally, the $\mathrm{CH}$ stars ( $\mathrm{HC}(3)$ sample) do have mean velocities which are twice the overall mean for the HC-sample they strongly affect. The mean distances to the Galactic plane are however similar for the three samples as found for mean true parallaxes. Conversely, the $\mathrm{HdC}$ and RCB stars (sample $\mathrm{HC}(2))$ display simultaneously smaller mean velocities and a stronger concentration to the Plane than stars of the whole HC-sample. Those latter two subsamples are however very small ( $n=23$ and 12 respectively) and the conclusions are only provisional.

The HC and CV-stars clearly belong to two distinct populations on kinematic grounds, as was the case for locations (Sect. 5). The differences are very large when the $\mathrm{CH}$ stars are included ( $\mathrm{HC}$ in Table 7$)$, and remain marked when they are rejected (sample $\mathrm{HC}(1))$. We have noticed that $\left\langle V_{\mathrm{t}}\right\rangle_{\mathrm{c}} \gtrsim\left\langle\left|V_{\mathrm{r}}\right|\right\rangle$ for the $\mathrm{CV}$-stars located on orbits close to the Galactic plane. This is also the case for the $\mathrm{HC}(1)$-sample which is supposed to be free of $\mathrm{CH}$ stars. Conversely, it is observed that $\left\langle V_{\mathrm{t}}\right\rangle_{\mathrm{c}} \simeq 2\left\langle\left|V_{\mathrm{r}}\right|\right\rangle$ for the HC- and $\mathrm{HC}(3)$-samples. The causes for those differences appear in the detailed analyses of Sects. 9 and 11.

Mean effective temperatures from Bergeat et al. (2001) and mean absolute bolometric magnitudes (Knapik et al. 2001) freed from the bias described by Smith \& Eichhorn (1996), and corrected for the influence of the Malmquist bias as described in Sect. 4, are quoted in Table 7. It is confirmed that the HdC and RCB stars are found in the high effective temperatures fringe of the HC-stars. No such trend is seen for the $\mathrm{CH}$ stars. No significant difference is found between the $\left\langle M_{\text {bol }}\right\rangle$-values of the three HC subsamples which are surprisingly close to one another.

\section{The reflex solar velocities for $\mathrm{HC}$ and $\mathrm{CV}$-stars}

\subsection{The unbiased angular velocities}

The basic considerations of the present section can be found in Mihalas \& Binney (1981, Sect. 6) and the used equations are given in Appendix A. We have adopted for the velocity components, the following usual frame: the $U$-axis towards the Galactic anticenter, the $V$-axis towards the direction of Galactic rotation, both axes lying 
in the Galactic plane, and the $W$-axis pointing towards the North Galactic Pole.

Calculating the mean values $\langle U\rangle,\langle V\rangle$ and $\langle W\rangle$ from Eqs. (A5) to (A7), we meet the same bias as described in Sect. 3.1. It originates from the very fact that the components are not linear functions of the true parallax $\varpi$. We use angular velocities instead, which are linear in $\varpi$, viz.

$\varpi U=f_{U}\left(l, b, \phi, \mu_{\alpha} \cos \delta, \mu_{\delta}\right)-\varpi V_{\mathrm{r}} \cos b \cos l=\nu_{U}(44)$

$\varpi V=f_{V}\left(l, b, \phi, \mu_{\alpha} \cos \delta, \mu_{\delta}\right)+\varpi V_{\mathrm{r}} \cos b \sin l=\nu_{V}(45)$

$\varpi W=f_{W}\left(l, b, \phi, \mu_{\alpha} \cos \delta, \mu_{\delta}\right)+\varpi V_{\mathrm{r}} \sin b=\nu_{W}$.

The mean values $\left\langle\nu_{U}\right\rangle,\left\langle\nu_{V}\right\rangle$ and $\left\langle\nu_{W}\right\rangle$ are thus unbiased. We may then derive the mean velocities from

$$
\begin{aligned}
\langle U\rangle & =\left\langle\nu_{U}\right\rangle /\langle\varpi\rangle \quad\langle V\rangle=\left\langle\nu_{V}\right\rangle /\langle\varpi\rangle \\
\langle W\rangle & =\left\langle\nu_{W}\right\rangle /\langle\varpi\rangle
\end{aligned}
$$

provided the velocities and true parallaxes are uncorrelated. This should in principle be the case since we are dealing with samples of relatively nearby stars distributed on the whole sky, with no marked correlation with the spiral-arm structure (Sect. 2 of Paper I).

We have however conducted various tests, such as the diagrams of $U, V$ and $W$ vs. $\varpi$ where the "rule of thumb" was applied and least square regressions as well. The former method may help in detecting possible failures in the latter one. No significant correlation was found.

\subsection{The main results for the $H C$ and $C V$-stars}

Making use of Eqs. (A8), (A11) and (A12), the solar velocities were derived for four samples (Table 8), (1) the CV-stars, (2) the HC-stars, (3) the HC-stars not confirmed as $\mathrm{CH}$ stars (HC'), and (4) the confirmed $\mathrm{CH}$ stars. Error estimates are also given. The latter were evaluated in a rough way, for various accuracies of estimated true parallaxes as quoted in Paper I, quoted errors on observed proper motion components (ESA 1997), and errors or quality quotation (a, b...) of observed radial velocities. The influence of relative errors on proper motions proves negligible. The influence of estimated true parallaxes is usually larger than that of radial velocities. When inaccurate, the latter may occasionally play a role. The degree of correlation, usually small, of the various terms in the Appendix equations, is difficult to estimate, especially for small samples corresponding to individual photometric groups.

The results are compared to the values derived by Dean (1976) from a sample (C) of 427 radial velocities. The majority of Dean's stars are CV-stars but some HCstars were included as well, which probably explain why his data seem intermediate between ours for the CVand HC-samples respectively. Its mean value of the distance to the Galactic plane is however $\langle|z|\rangle \simeq 0.213 \mathrm{kpc}$ which is practically our value $(0.22 \pm 0.03)$ for the CVstars (Table 7). The same is true for the stellar residualvelocities distributions (see Sect. 11 and Table 11), except
Table 8. Solar velocities in $\mathrm{km} \mathrm{s}^{-1}$ and apex directions (galactic coordinates in ${ }^{\circ}$ ) for our four samples (S) of carbon stars. In the second zone, the results from Dean (1976) for a mixed sample (C), and those of Hartwick \& Cowley (1985) for $\mathrm{CH}$ stars $(\mathrm{CH})$. Reference solar motions are given in the third zone.

\begin{tabular}{ccccccccc}
\hline $\mathrm{S}$ & $n$ & $u_{\odot}$ & $v_{\odot}^{\prime}$ & $v_{1}$ & $w_{\odot}$ & $S_{\odot}$ & $l_{\mathrm{A}}$ & $b_{\mathrm{A}}$ \\
\hline $\mathrm{CV}$ & 180 & -2.3 & 5.85 & -0.6 & 4.3 & 7.6 & 68 & 34 \\
& & \pm 2.6 & \pm 2.1 & & \pm 1.8 & \pm 2.1 & \pm 24 & \pm 20 \\
$\mathrm{HC}$ & 90 & -16.3 & 30.5 & -25.3 & 6.5 & 35.2 & 62 & 11 \\
& & \pm 3.6 & \pm 3.2 & & \pm 2.4 & \pm 3.3 & \pm 6 & \pm 4 \\
$\mathrm{HC}$ & 70 & -14.6 & 11.0 & -5.7 & 3.8 & 18.65 & 37 & 12 \\
& & \pm 4.0 & \pm 3.1 & & \pm 2.7 & \pm 3.7 & \pm 11 & \pm 9 \\
$\mathrm{CH}$ & 20 & -29 & 117 & -112 & 21 & 122 & 76 & 10 \\
& & \pm 10 & \pm 10 & & \pm 7 & \pm 10 & \pm 5 & \pm 4 \\
\hline $\mathrm{C}$ & 427 & -9.6 & 11.7 & -6.5 & 4.6 & 15.8 & 51 & 17 \\
& & \pm 2.0 & \pm 2.2 & & \pm 3.2 & \pm 2.2 & \pm 8 & \pm 12 \\
$\mathrm{CH}$ & 51 & -1 & 147 & -142 & 9 & 147 & 90 & 3.5 \\
& & \pm 37 & \pm 35 & & \pm 24 & \pm 35 & \pm 15 & \pm 9 \\
\hline $\mathrm{SSM}$ & & -10.4 & 14.8 & -9.6 & 7.3 & 19.5 & 56 & 23 \\
$\mathrm{BSM}$ & & -9 & 11 & -5.75 & 6 & 15.4 & 51 & 23 \\
$\mathrm{PSV}$ & & -9 & 12 & -6.75 & 7 & 16.5 & 53 & 25 \\
$\mathrm{SLS}$ & & -10.0 & 5.25 & 0.0 & 7.17 & 13.4 & 28 & 32 \\
& & \pm 0.4 & \pm 0.6 & & \pm 0.4 & \pm 0.4 & \pm 3 & \pm 2 \\
\hline
\end{tabular}

for the component perpendicular to the Galactic plane which is slightly smaller than ours for the CV-sample.

We have added in Table 8 the results for the standard solar motion (SSM) and for the basic solar motion (BSM) as given by Mihalas \& Binney (1981, p. 398). The components of the peculiar velocity of the Sun relative to the LSR (namely PSV) from Mihalas \& Binney (1981, p. 400) are also given. We however adopt the values $\left(\mathrm{km} \mathrm{s}^{-1}\right)$

$u_{\odot} \simeq-10.0 \quad v_{\odot} \simeq 5.25 \quad w_{\odot} \simeq 7.17$

as derived by Dehnen \& Binney (1998) from HIPPARCOS kinematics of 11865 main sequence stars (SLS). The analysis of the distribution of nearby stars in the velocity space inferred from HIPPARCOS data on 14369 stars can be found in Dehnen (1998). The azimuthal component is smaller $\left(5.2 \mathrm{~km} \mathrm{~s}^{-1}\right)$ than the classical value of $\left(12 \mathrm{~km} \mathrm{~s}^{-1}\right)$. This latter feature is common to recent studies (see the references in Dehnen \& Binney 1998). The $u_{\odot}-$ values in Table 8 are negative ones while those of $w_{\odot}$ are all positive ones. This is also true for the values quoted in Tables 6-3 of Mihalas \& Binney (p. 396) for various groups of stars. These components are directed respectively towards the Galactic center and the North Galactic Pole. The $v_{\odot}^{\prime}$-components are all positive in Table 8 as it is the case in the table of Mihalas \& Binney. From Eqs. (A10) and (48), it happens that

$v_{1} \simeq 5.25-v_{\odot}^{\prime}$

the values being given in Col. 5 of Table 8 . We note an appreciable lag of the HC-sample with respect to the Sun $\left(v_{1} \simeq-25.3 \mathrm{~km} \mathrm{~s}^{-1}\right)$ which is essentially due to the $\mathrm{CH}$ stars since the value for the HC'-sample is much smaller $(-5.7)$. It is well known that the Galactic thick disk is rotating rapidly (e.g. Freeman 1987a, Sect. 3; Majewski 1993, Sect. 3.3.1 and Fig. 6). The asymmetric drift increases with median $|z|$, with however high dispersion in 
the values from various tracers of the thick disk. At about $0.5 \mathrm{kpc}$ (which is the case of the HC-sample: see Table 7), the estimates range from 0 to $-60 \mathrm{~km} \mathrm{~s}^{-1}$, while drifts of -30 to $-110 \mathrm{~km} \mathrm{~s}^{-1}$ are observed beyond $1 \mathrm{kpc}$.

The same lag is responsible for $S_{\odot} \simeq 35.2 \mathrm{~km} \mathrm{~s}^{-1}$ for the HC-group, while $S_{\odot} \simeq 18.7 \mathrm{~km} \mathrm{~s}^{-1}$ for the HC'-group is close to the values for SSM and BSM. The apex coordinates are roughly consistent for the five groups considered. The values of $u_{\odot}, v_{\odot}^{\prime}, w_{\odot}$ and $S_{\odot}$ for $\mathrm{CV}$-stars are all small ones. There is no appreciable lag or leading of the CV-sample with respect to the Sun. These results confirm the CV-groups belonging to a classic disk population (or thin disk). The HC-groups seem again representative of an older kinematic population (thick disk). It is contaminated by a spheroidal component, the $\mathrm{CH}$ stars, which is similar to the sample of Hartwick \& Cowley (1985).

\subsection{The trends along the photometric sequence of groups}

Bergeat et al. (2001) have shown that the sequence of photometric groups from $\mathrm{HC} 0$ to $\mathrm{HC} 5$ and then from $\mathrm{CV} 1$ to CV7 is a sequence of decreasing effective temperatures. It is also a sequence of increasing luminosities as shown by Knapik (1999) and Knapik et al. (2001). The mean values displayed in Table 3 illustrate this result. The analysis of the kinematic trends along this sequence is thus tempting, but limited by low accuracy.

The average solar velocities and apex coordinates as obtained for 11 of the 14 photometric groups are given in Table 9. A clear-cut dichotomy between the HC- and CVgroups, is confirmed. The solar space velocity drops from more than $30 \mathrm{~km} \mathrm{~s}^{-1}$ to $7-15 \mathrm{~km} \mathrm{~s}^{-1}$, and the jump seems to occur between HC5 and CV1.

Since the $\mathrm{CH}$ stars are considered as halo tracers, we also computed the solar velocities in new HC-samples without the confirmed CH stars (star-symbols in Table 9). No confirmed $\mathrm{CH}$ star was found in the CV-groups. The most outstanding result is that the HC-like velocity data of HC5 is entirely due to one star, namely C327 = V Ari, and that the $\mathrm{HC} 5{ }^{*}$-results are seemingly $\mathrm{CV}$-like. A ratio of 2.7 however remains between the mean solar velocities of the $\mathrm{HC}^{*}{ }^{*}$ - to $\mathrm{HC}^{*}{ }^{*}$-groups

$\left\langle S_{\odot}\right\rangle \simeq 29.1 \pm 2.6$

and those of the $\mathrm{HC} 5{ }^{*}$ - and $\mathrm{CV}$-groups

$$
\left\langle S_{\odot}\right\rangle \simeq 10.7 \pm 1.2
$$

significant at more than $4 \sigma$. The latter value is typical of the intermediate and old (dwarf) population of the thin disk while the former one points to older stars (e.g. Mihalas \& Binney 1981), of the thick disk population.

Revisiting the data on mean distances to the Galactic plane, we could see that despite the HC5 high value $\langle|z|\rangle_{\mathrm{c}} \simeq 0.46 \mathrm{kpc}$ (see Table 2), the poorly-documented distribution $N(|z|)$ seems finally CV-like. Removing V Ari
Table 9. Solar velocities in $\mathrm{km} \mathrm{s}^{-1}$ and apex directions for 11 photometric groups of carbon stars (same meanings as in Table 8). The star symbol refers to samples without confirmed spectroscopic $\mathrm{CH}$ stars. It can be seen that HC5* (V Ari removed) is closer to the CV-groups than to the other $\mathrm{HC}$ ones.

\begin{tabular}{|c|c|c|c|c|c|c|c|c|}
\hline $\mathrm{G}$ & $i$ & $n$ & $u_{\odot}$ & $v_{\odot}^{\prime}$ & $w_{\odot}$ & $S_{\odot}$ & $l_{\mathrm{A}}$ & $b_{\mathrm{A}}$ \\
\hline \multirow[t]{2}{*}{$\mathrm{HC} 1$} & 1 & 23 & 6 & 28 & 27 & 39 & -78 & 43 \\
\hline & & & \pm 7 & \pm 7 & \pm 5 & \pm 6 & \pm 15 & \pm 13 \\
\hline \multirow[t]{2}{*}{$\mathrm{HC} 1 *$} & 1 & 18 & -7 & 25 & 14 & 30 & 75 & 28 \\
\hline & & & \pm 8 & \pm 7 & \pm 6 & \pm 7 & \pm 18 & \pm 14 \\
\hline \multirow[t]{2}{*}{$\mathrm{HC} 2$} & 2 & 25 & -43 & 69 & 2 & 82 & 58 & 1.4 \\
\hline & & & \pm 7 & \pm 6 & \pm 5 & \pm 7 & \pm 5 & \pm 3 \\
\hline \multirow[t]{2}{*}{$\mathrm{HC} 2 *$} & 2 & 18 & -27 & 24 & 0.9 & 36 & 42 & 1.4 \\
\hline & & & \pm 8 & \pm 7 & \pm 6 & \pm 8 & \pm 12 & \pm 9 \\
\hline \multirow[t]{2}{*}{ HC3 } & 3 & 14 & -18 & -11 & -13 & 25 & -33 & -31 \\
\hline & & & \pm 9 & \pm 8 & \pm 6 & \pm 8 & \pm 24 & \pm 21 \\
\hline \multirow[t]{2}{*}{$\mathrm{HC}^{*}$} & 3 & 12 & -18 & -15 & -6 & 24 & -41 & -14 \\
\hline & & & \pm 10 & \pm 9 & \pm 7 & \pm 10 & \pm 23 & \pm 18 \\
\hline \multirow[t]{2}{*}{$\mathrm{HC} 4$} & 4 & 12 & -6 & 24 & 31 & 39 & 77 & 51 \\
\hline & & & \pm 10 & \pm 9 & \pm 7 & \pm 8 & \pm 23 & \pm 21 \\
\hline \multirow[t]{2}{*}{$\mathrm{HC} 4 *$} & 4 & 7 & -15 & 0.1 & 22 & 27 & 0.2 & 56 \\
\hline & & & \pm 13 & \pm 12 & \pm 9 & \pm 11 & \pm 45 & \pm 48 \\
\hline \multirow[t]{2}{*}{ HC5 } & 5 & 16 & -13 & 29 & -17 & 36 & 66 & 28 \\
\hline & & & \pm 9 & \pm 8 & \pm 6 & \pm 8 & \pm 15 & \pm 13 \\
\hline \multirow[t]{2}{*}{ HC5* } & 5 & 15 & -6 & 4 & -6 & 9 & 33 & 36 \\
\hline & & & \pm 9 & \pm 8 & \pm 6 & \pm 8 & \pm 63 & \pm 58 \\
\hline \multirow[t]{2}{*}{ CV1 } & 6 & 29 & -6 & 9 & -6 & 13 & 56 & 27 \\
\hline & & & \pm 7 & \pm 5 & \pm 4 & \pm 5 & \pm 31 & \pm 25 \\
\hline \multirow[t]{2}{*}{ CV2 } & 7 & 43 & -0.4 & 7 & 3 & 7 & 86 & 22 \\
\hline & & & \pm 5 & \pm 4 & \pm 4 & \pm 4 & \pm 45 & \pm 34 \\
\hline \multirow[t]{2}{*}{ CV3 } & 8 & 30 & -9 & 6 & 12 & 16 & 33 & 48 \\
\hline & & & \pm 6 & \pm 5 & \pm 4 & \pm 5 & \pm 29 & \pm 30 \\
\hline \multirow[t]{2}{*}{ CV4 } & 9 & 28 & -6 & 2 & 2 & 7 & 19 & 15 \\
\hline & & & \pm 7 & \pm 5 & \pm 5 & \pm 6 & \pm 48 & \pm 42 \\
\hline \multirow[t]{2}{*}{ CV5 } & 10 & 27 & 2 & 8 & 5 & 9 & -74 & 30 \\
\hline & & & \pm 7 & \pm 5 & \pm 5 & \pm 4 & \pm 47 & \pm 35 \\
\hline \multirow[t]{2}{*}{ CV6 } & 11 & 24 & 7 & 2 & 12 & 14 & -15 & 57 \\
\hline & & & \pm 7 & \pm 6 & \pm 5 & \pm 6 & \pm 45 & \pm 52 \\
\hline HC1* & 1.5 & 36 & -17 & 25 & 7 & 31 & 56 & 14 \\
\hline$+\mathrm{HC} 2 *$ & & & \pm 6 & \pm 5 & \pm 4 & \pm 5 & \pm 11 & \pm 8 \\
\hline HC3* & 3.4 & 19 & -17 & -10 & 4 & 20 & -30 & 13 \\
\hline$+\mathrm{HC} 4 *$ & & & \pm 8 & \pm 7 & \pm 6 & \pm 8 & \pm 22 & \pm 17 \\
\hline
\end{tabular}

only reduces $\langle|z|\rangle_{\mathrm{c}}$ to 0.44 . We conclude that the space distribution and the kinematics of $\mathrm{HC} 5^{*}$ are typically $\mathrm{CV}$ like, but that the populated galactic volume (i.e. the galactic scale above the plane) is rather HC-like. Between mean values, a difference was found at $3 \sigma$, on the first coordinate, with for the $\mathrm{HC}^{*}$ to $\mathrm{HC}^{*}{ }^{*}$ sample

$\left\langle u_{\odot}\right\rangle \simeq-16.7 \pm 4.1$

while for the $\mathrm{HC}^{*}$ and $\mathrm{CV}$-groups

$\left\langle u_{\odot}\right\rangle \simeq 3.0 \pm 2.8$.

Then we plotted against the $i$-index of the photometric groups, the $\left\langle u_{\odot}\right\rangle$-components (Fig. 6) and space velocities $\left\langle S_{\odot}\right\rangle$ (Fig. 7 ) to help in deciding what is real. Since the HC-stars are less numerous than the CV-ones, we introduced samples $\mathrm{HC}^{*}+\mathrm{HC}^{*}$ and $\mathrm{HC}^{*}+\mathrm{HC}^{*}$, as quoted in Table 9 . This choice is justified by compatibilities in absolute magnitudes and effective temperatures, and the will to keep $\mathrm{HC} 5^{*}$ as a separate group. The latter is found indistinguishable from CV1 in both figures. 


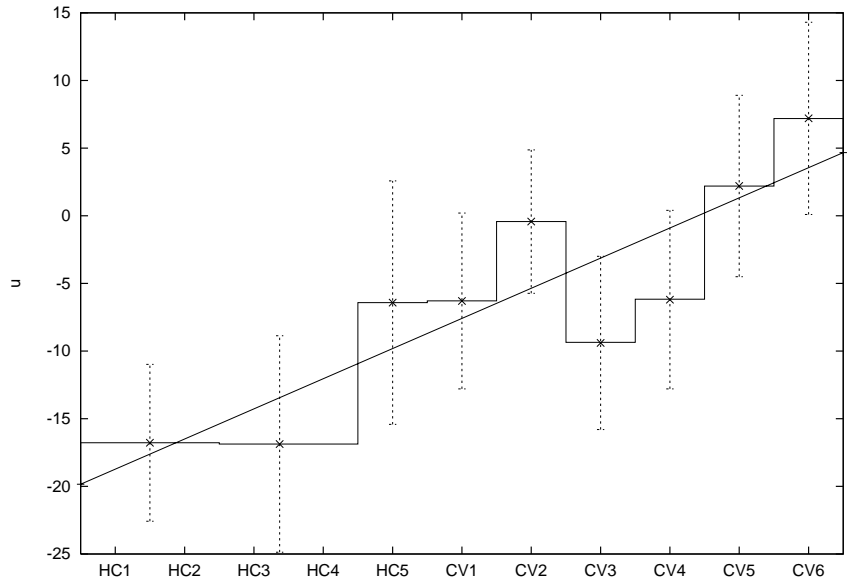

Fig. 6. The $u_{\odot}$-components of the solar reflex velocity for the photometric groups (data from Table $9 ; \mathrm{HC}^{*}$ 's samples).

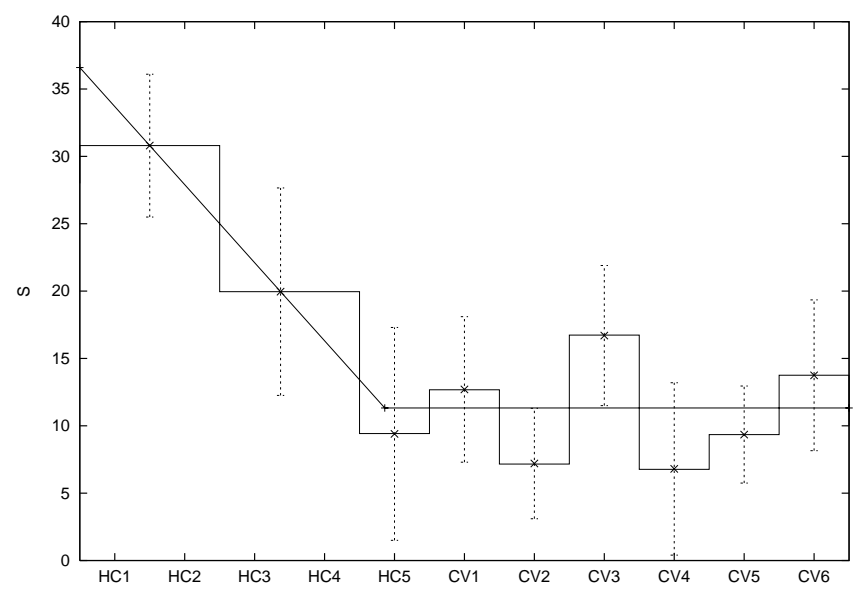

Fig. 7. The $S_{\odot}$-components of the solar reflex velocity for the photometric groups (data from Table $9 ; \mathrm{HC} j{ }^{*}$ 's samples).

The first plot shows a more or less regular gradient from negative values to a slightly positive one, according to

$u_{\odot} \simeq(2.23 \pm 0.49) i-(20.96 \pm 4.33) \quad \rho^{2} \simeq 0.75$.

Following the sequence of groups back from CV6 to HC1, we deal with stars moving more and more predominantly outward with respect to the Sun, and thus relative to the LSR. The diagram is not suggestive of a sharp discontinuity at $\mathrm{HC}^{*}{ }^{*}-\mathrm{HC} 5 *$, but larger samples are needed before a firm conclusion can be ascertained. The plot of $S_{\odot}$ vs. the $i$-index (Fig. 7) is more conclusive in this respect and a jump at $\mathrm{HC} 4{ }^{*}-\mathrm{HC} 5 *$ could be present with a flat portion from $\mathrm{HC} 5 *$ to $\mathrm{CV} 6$ indicated. We tentatively fit the distribution for the HC-groups by

$S_{\odot} \simeq-5.80 i+39.5$

and the HC5*-CV sequence by Eq. (51).

The solar reflex velocities toward the Galactic anticenter $\left(u_{\odot}\right)$ increase along the $\mathrm{HC}-\mathrm{CV}$ sequence of photometric groups, from negative values (i.e. directed towards Galactic center) to slightly positive ones. From radial velocity measurements of 179 carbon stars towards
Table 10. Solar velocities in $\mathrm{km} \mathrm{s}^{-1}$ and apex directions for carbon stars of various variability classes. The meanings are the same as in Table 8. The subsamples are: Lb (Irregulars); $\mathrm{SRb}$ (Semi-regulars of low amplitude); Miras (carbon Miras); M+SRa (Miras + Semi-regulars of large amplitude); LPV1 (Long period variables of $P<300^{\mathrm{d}}$ ); LPV2 (Long period variables of $P>300^{\mathrm{d}}$ ); Cst (photometric constants); Cst* (photometric constants without $19 \mathrm{CH}$ stars).

\begin{tabular}{ccccccccc}
\hline $\mathrm{G}$ & $n$ & $u \odot$ & $v_{\odot}^{\prime}$ & $v_{\mathrm{l}}$ & $w_{\odot}$ & $S_{\odot}$ & $l_{\mathrm{A}}$ & $b_{\mathrm{A}}$ \\
\hline $\mathrm{Lb}$ & 86 & -0.2 & 14 & -8.7 & -1.0 & 14 & 89 & -4 \\
& & \pm 4 & \pm 3 & & \pm 3 & \pm 3 & \pm 15 & \pm 11 \\
$\mathrm{SRb}$ & 77 & -4.9 & 3.5 & 1.8 & 6.8 & 9.0 & 35 & 49 \\
& & \pm 4 & \pm 3 & & \pm 3 & \pm 3 & \pm 34 & \pm 35 \\
Miras & 29 & 1.9 & 5.2 & 0.0 & 9.0 & 11 & -71 & 58 \\
& & \pm 7 & \pm 5 & & \pm 4 & \pm 5 & \pm 66 & \pm 62 \\
M+SRa & \multirow{2}{*}{43} & 1.0 & 2.7 & 2.5 & 7.9 & 8.5 & -71 & 70 \\
& & \pm 5 & \pm 4 & & \pm 4 & \pm 4 & \pm 102 & \pm 98 \\
LPV 1 & 56 & -6.0 & 2.9 & 2.3 & 5.7 & 8.8 & 26 & 41 \\
& & \pm 5 & \pm 4 & & \pm 3 & \pm 4 & \pm 34 & \pm 35 \\
LPV 2 & 54 & 1.3 & 3.7 & 1.6 & 12 & 13 & -71 & 73 \\
& & \pm 5 & \pm 4 & & \pm 3 & \pm 3 & \pm 68 & \pm 66 \\
Cst & \multirow{2}{*}{61} & -18 & 42 & -37 & 13 & 48 & 67 & 15 \\
& & \pm 5 & \pm 4 & & \pm 3 & \pm 4 & \pm 6 & \pm 4 \\
Cst* & \multirow{2}{*}{42} & -11 & 24 & -19 & 6.9 & 27 & 66 & 15 \\
& & \pm 5 & \pm 5 & & \pm 4 & \pm 5 & \pm 11 & \pm 8 \\
\hline
\end{tabular}

the Galactic anticenter, Metzger \& Schechter (1994) already found that their sample is moving radially outward with respect to the LSR at a velocity of $(6.6 \pm 1.7) \mathrm{km} \mathrm{s}^{-1}$, i.e. corresponding to $u_{\odot} \simeq-6.6$ which is very close to our average $u_{\odot} \simeq-(7.0 \pm 1.0) \mathrm{km} \mathrm{s}^{-1}$ for 270 carbon stars. There is actually no reason to favor linear relations and curved fits might prove better for $u_{\odot}$ and $S_{\odot}$ when more accurate data will be available. Clearly more data is needed before a firm conclusion can be reached. Intruders from the halo may well remain in the $\mathrm{HC}^{*}$-samples.

Dehnen (2000) studied the HIPPARCOS data for 3527 main-sequence stars with $B-V \geq 0.6$ and 2491 mainly late-type non-main-sequence stars, high velocity stars excluded. In a $(u, v)$-diagram (velocities relative to LSR and u-axis towards Galactic center: his Fig. 9, p. 809), he found a bimodal distribution he attributed to the effect of the second Lindblad resonance. Our data (after subtraction of the solar motion relative to LSR of Dehnen \& Binney 1998) coincide with his principal mode (essentially his sample of early-type stars, including the Sun). No coincidence was found with his secondary mode (intermediate velocities) mainly populated by late-type stars.

\section{Reflex solar velocity vs. variability classes}

We have considered samples delineated according to the classification scheme of the GCVS catalog of Kholopov et al. (1985). Again the components of the reflex solar velocity were derived from the method used in Sect. 9. The results are quoted in Table 10. The distribution of the carbon stars in terms of photometric groups is shown in Fig. 8 for every variability class (M for carbon Miras, SRa for large amplitude semi-regular variables, SRb for smaller amplitude semi-regulars, Lb for irregular variables and Cst 


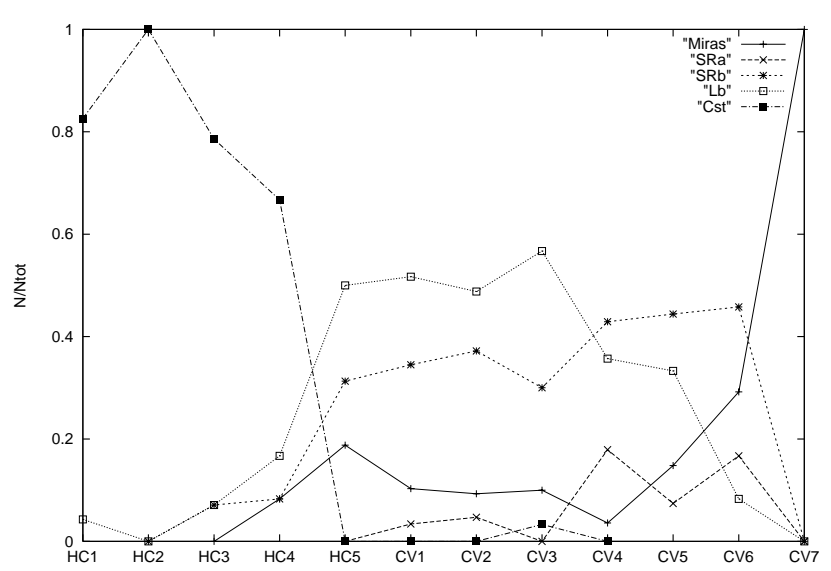

Fig. 8. The distribution of the 276 HIPPARCOS carbon stars with variability class amongst the photometric groups, normalized to unity.

for constant stars). It appears that the constant stars are also HC-stars while most of the variable carbon stars are found in the CV-groups as first-intended. Also, the Lbvariables are predominantly HC5 to CV3, which makes them intermediate. Then, the $\mathrm{SRb}$-variables are abundant from HC5 to CV6, while the SRa-variables and Miras appear as more concentrated between CV4 and CV7. There is a statistical correlation between the sequence of increasing variability "Cst, Lb, SRb, SRa and Miras" and the sequence of photometric groups "HC1 to CV7". This point will be further discussed in Knapik et al. (2001), together with mean luminosities.

The sample of the irregular (Lb) variables is the largest one. It displays a slight lag in $v_{\odot}^{\prime}$, which makes it again intermediate between the other variables on one hand and the constant stars on the other hand. While the former have practically no lag like the CV-stars, the latter show an appreciable lag like the HC-stars (Table 8 and Table 10). The compositions of variability classes as shown in Fig. 8, explain those results. The Lband SRb-samples contain 2 and $3 \mathrm{CH}$ stars respectively. Removing them does not change appreciably the results of Table 10). Conversely, a Cst*-sample is considered from which $19 \mathrm{CH}$ stars were rejected. The lag $v_{\mathrm{l}}$ and the spatial velocity $S_{\odot}$ are almost halved in this latter sample.

We also considered two subsamples, namely LPV 1 with $P<300^{\mathrm{d}}$ and LPV 2 with $P>300^{\mathrm{d}}$. The latter is dominated by Miras and SRas and the solution found is consistent. The solar velocity for the LPV 1 sample is close to the result for the SRb-sample. The trend to larger components of the solar reflex velocity for shorter periods, as shown by oxygen-rich LPVs (e.g. Delhaye 1965; Mihalas \& Binney 1981, Table 6.3 p. 396), is not found here. Those latter giants presumably correspond to the second mode (Sect. 9) of Dehnen (2000).

\section{The ellipsoids of residual velocities}

We finally study the stellar residual-velocity distributions, determining their axes and maximal dispersions, and then the dispersions along the three galactic axes. We make
Table 11. The extreme dispersions $\sigma_{j}$ and their directions given as galactic longitudes $L_{j}$ and latitudes $B_{j}$, for the samples of Table 8 . The estimated dispersions along the Galactic axes (last three columns) are compared to published values: a This paper; ${ }^{\mathrm{b}}$ Dean (1976); ${ }^{\mathrm{c}}$ Hartwick \& Cowley (1985); ${ }^{d}$ Old Disk (Freeman 1987a); ${ }^{\text {e }}$ Thick Disk (Norris 1999). We found in every case $\sigma_{w} \simeq \sigma_{3}$.

\begin{tabular}{|c|c|c|c|c|c|c|c|c|}
\hline $\mathrm{S}$ & $n$ & $j$ & $L_{j}$ & $B_{j}$ & $\sigma_{j}$ & $\sigma_{u}$ & $\sigma_{v}$ & $\sigma_{w}$ \\
\hline \multirow[t]{3}{*}{$\mathrm{CV}^{\mathrm{a}}$} & 180 & 1 & 5.3 & 1.9 & 30.2 & 30.1 & 22.2 & 23.4 \\
\hline & & 2 & 93.8 & 38.3 & 22.1 & & & \\
\hline & & 3 & 277.7 & 51.6 & 23.4 & & & \\
\hline $\mathrm{C}^{\mathrm{b}}$ & 427 & & & & & 29.8 & 19.8 & 14.0 \\
\hline$O D^{d}$ & & & & & & 40 & 25 & 20 \\
\hline \multirow[t]{3}{*}{$\mathrm{HC}^{\mathrm{a}}$} & 90 & 1 & 74.0 & 15.0 & 106.0 & 85.4 & 104.3 & 54.4 \\
\hline & & 2 & 343.2 & -2.9 & 83.3 & & & \\
\hline & & 3 & 62.4 & -74.7 & 54.4 & & & \\
\hline \multirow[t]{3}{*}{$\mathrm{HC}^{\mathrm{a}}$} & 70 & 1 & 328.5 & 15.4 & 59.6 & 59.0 & 57.8 & 42.1 \\
\hline & & 2 & 56.4 & 8.0 & 57.0 & & & \\
\hline & & 3 & 300.5 & -72.7 & 42.3 & & & \\
\hline $\mathrm{TD}^{\mathrm{e}}$ & & & & & & 65 & 54 & 38 \\
\hline \multirow[t]{3}{*}{$\mathrm{CH}^{\mathrm{a}}$} & 20 & 1 & 756 & 17 & 281 & 163 & 277 & 85 \\
\hline & & 2 & 349 & 12 & 156 & & & \\
\hline & & 3 & 293 & 68 & 86 & & & \\
\hline $\mathrm{CH}^{\mathrm{c}}$ & 51 & & & & & $?$ & 161 & 125 \\
\hline
\end{tabular}

use of a method adapted from Charlier (1926), already adopted by Bergeat et al. (1978). An account of Charlier's method can be found in Atanasijević (1971).

We abandoned the idea of a detailed study of the residual-velocity distributions in each photometric group, due to small samples and low accuracy. The results are summarized in Table 11, where the dispersions are significantly higher for the HC-sample than for the CV-sample. The ratio ranges from 2.3 (third axis) to about 4 (first and second axes which are nearly in the Galactic plane). Removing the confirmed CH stars, we obtain the HC'sample whose velocity dispersions are about twice those of the CV-sample, whichever axis is considered. Those results again point to two distinct populations, the $\mathrm{HC}$ one being contaminated by a contribution from halo ( $\mathrm{CH}$ stars). The vertical dispersion of 42 to $54 \mathrm{~km} \mathrm{~s}^{-1}$ observed for the HC'- and HC-samples, is typical of the thick disk (40-50 $\mathrm{km} \mathrm{s}^{-1}$, Freeman 1987a; Gilmore 1989) while the value of $23 \mathrm{~km} \mathrm{~s}^{-1}$ for the $\mathrm{CV}$-sample is representative of the old (thin) disk $\left(20 \mathrm{~km} \mathrm{~s}^{-1}\right.$; Gilmore 1989). More specifically, the three velocity components usually adopted for the thick disk from Norris (1999) and for the old disk from Freeman (1987a) are given for comparison with the values for the HC'- and CV-samples respectively. The agreement is excellent in both cases. As usual, it is found that the third axis (smallest extreme dispersion) of the velocity ellipsoid is oriented nearly perpendicular to the Galactic plane, and the other two axes lie approximately in the Plane. Usually, the direction of the largest velocity dispersion is nearly toward the Galactic center. This is very nearly the case of the CV-sample.

Making use of the age versus velocity (AVR) relation of Wielen et al. (1992), the $\sigma \simeq 44 \mathrm{~km} \mathrm{~s}^{-1}$ obtained for the space velocities of CV-stars of Table 11 would correspond to an average of $(3 \pm 1)$ Gyr. Within the uncertainties, the 
AVR of Carlberg et al. (1985) would yield (6 41.5$)$ Gyr. The two relations actually differ. From an analysis of the HR diagram and pulsation masses (Knapik et al. 2001), we favor the former age but there is probably a considerable spread in individual values from a few $10^{2} \mathrm{Myr}$ up to 8-12 Gyr. The values for the HC-groups fell completely outside the AVRs published in both papers. It is clear again that the HC-stars are a distinct older population with large velocity dispersions. The various lags of $\mathrm{CH}$ stars along the $v$-axis influence the dispersions in the Galactic plane, and deviate the vertex axis.

\section{Discussion and conclusion}

The space distribution and velocity data of HIPPARCOS carbon-rich giants were studied in detail. The biases (Lutz-Kelker, Smith-Eichhorn and Malmquist) were investigated, refining the analysis of Paper I. While the LutzKelker bias (1973) is accounted for in our estimated true parallaxes, the bias described by Smith \& Eichhorn (1996) is avoided, as far as possible, by averaging on quantities which are linear functions of parallaxes. Corrections for mean distances and absolute magnitudes were proposed to account for the Malmquist bias $(1924,1936)$. They prove to be small here. The exponent in the frequency distribution of true parallaxes is however increased from -2.9 or $-3 \pm 0.15$ (uniform slab) to $-2.35 \pm 0.10$ by the Malmquist bias. The HIPPARCOS sample seems almost complete up to a distance of nearly $1 \mathrm{kpc}$ from the sun. It was shown that the average height above the Plane is $(0.50 \pm 0.06) \mathrm{kpc}$ for the HC-stars, about three times that of the CV-stars, viz. $(0.16 \pm 0.03) \mathrm{kpc}$.

The vertical space distributions are described in terms of a simple exponential law with a distance scale for HCstars much larger $(0.95 \pm 0.06 \mathrm{kpc}$, thick disk $)$ than that for the CV-stars $(\simeq 0.19 \mathrm{kpc}$, thin disk). The local densities in the Plane and projected surface densities were deduced, with an estimated total of nearly $76 \mathrm{kpc}^{-2}$, among which $29 \%$ are HC-stars. If the HC5-stars (whose case is not clear) are rejected, this percentage falls to $24 \%$ with $18 \%$ attributed to thick disk HC-stars and $6 \%$ to halo $\mathrm{CH}$ stars. Detailed densities were established in terms of photometric groups and effective temperatures, confirming asymmetric and bimodal distributions (HC- and CV-zones).

The mean radial and tangential velocities with respect to the Sun turn out to be about $25 \mathrm{~km} \mathrm{~s}^{-1}$ for $195 \mathrm{CV}$-stars and about $50 \mathrm{~km} \mathrm{~s}^{-1}$ for 81 non-CH HCstars, that is an unambiguous ratio of 2 , suggestive of distinct kinematic populations. Those average velocities are much higher for 23 confirmed $\mathrm{CH}$ stars, in excess of $100 \mathrm{~km} \mathrm{~s}^{-1}$. The reflex solar velocity with respect to our groups was then calculated and compared to reference motions. Also they are quite consistent with previous results for carbon stars. While the $\mathrm{CH}$ stars show halo velocities with a substantial drift of $-112 \mathrm{~km} \mathrm{~s}^{-1}$ in the $v$ velocity, typical of a slowly rotating system (Hartwick \& Cowley found $-135 \mathrm{~km} \mathrm{~s}^{-1}$ ), the contaminated HC-sample shows only $-25.3 \mathrm{~km} \mathrm{~s}^{-1}$. In the case of the HC'-sample, it is even smaller $\left(-5.7 \mathrm{~km} \mathrm{~s}^{-1}\right)$, i.e. in the low-range drift velocity of thick disk tracers (Majewski 1993) with median distances from plane of about $0.5 \mathrm{kpc}$. No appreciable lag is found for the CV-stars $\left(-0.6 \pm 2 \mathrm{~km} \mathrm{~s}^{-1}\right)$, whose space distributions and velocities are typical of the thin disk, most of them being younger and more massive than the Sun. Large velocities perpendicular to the Plane are observed mostly for the $\mathrm{CH}$ stars. The $u_{\odot}$ component increases along the sequence of photometric groups. Correspondingly, the space velocity $S_{\odot}$ appears as 2.7 times larger for $\mathrm{HC}^{*}$-stars than it is for $\mathrm{CV}$-stars.

The analyses according to variability classes illustrate the fact that variables are essentially in the CV-groups while constant stars are found in the HC-groups. Amongst the carbon variables, the irregular variables (Lb-class) exhibit kinematic data (lag and space velocity), intermediate between the other variables and the constant stars.

Finally, the ellipsoids of the residual velocities of HCand CV-stars were investigated. The dispersions of the HC sample range from 2.3 to 4 times higher than those of the CV-sample. The ratio still amounts to about 2 when the non-CH HC stars (i.e. the HC'-sample) are considered. Their dispersions are remarkably close to the values quoted by Norris (1999) for the bulk thick disk stars. The $\sigma_{w} \simeq 40 \mathrm{~km} \mathrm{~s}^{-1}$ value is characteristic of the thick disk population at the solar distance from the Galactic center (Freeman 1987a, p. 612). The dispersions of the CVsample are very close to the values usually quoted for the old disk (Freeman 1987a). Making use of the age vs. velocity relations (AVR) of Wielen et al. (1992), we obtained 3 Gyr as a typical average age of the CV-sample, and $6 \mathrm{Gyr}$ from the relation of Carlberg et al. (1985), which seems less likely. The individual values probably range from a few hundred million years up to 8-12 Gyr. The HC and HC'-samples clearly fall outside those relations, and lowmass stars with ages in excess of $10 \mathrm{Gyr}$ are indicated.

We conclude that the $\mathrm{CV}$-sample is a component of the old (thin) disk. Most HC-stars are members of the thick disk with contaminations from halo $\mathrm{CH}$ stars and a few CV-stars. Conversely, the Mira V CrB (CV7) might be a halo intruder on the basis of its high velocities, especially perpendicular to Plane. The thick-disk membership on the basis of space distribution and velocities, and the high space density of HC-stars, are certainly important clues in the investigation of the true nature of the mysterious hot carbon stars, which have lower metallicities on average (e.g. Wallerstein \& Knapp 1998). Less dragged-up carbon is needed to make a carbon star from a star with low initial $\mathrm{O} / \mathrm{H}$ ratio, and low mass stars do have smaller hydrogenrich envelopes to enrich of only a few tenths of one solar mass. Possible correlations of kinematics and metallicities need be investigated (see Prochaska et al. 2000 for stellar abundances in the thick disk).

The HC-stars are relatively low-luminosity objects with $\left\langle M_{\text {bol }}\right\rangle \simeq-1.8$ (Tables $3 \& 7$ ). The third Dredgeup process of carbon and (eventually) s-process elements in TP-AGB stars (Iben \& Renzini 1983) occurs only for $M_{\mathrm{bol}} \leq-3.5 \pm 0.5$, depending on details of the models 
and codes used (e.g. Marigo et al. 1999). Those models fail to explain the HC-stars. Full discussion of this topic is postponed to a future paper (Knapik et al. 2001). For the time being, we emphasize the point that the high frequency of the thick disk HC'-stars $(\geq 18 \%)$ is a strong argument against theories necessitating rare events. The binary model itself, for which no evidence was found in the observations of HC'-stars (McClure 1997a), seems unlikely. The discrepancy possibly originates in insufficient knowledge of mixing processes in low-mass stars evolving along RGB and AGB or at He-flash, prior to TP-AGB (Busso et al. 1999).

Suntzeff et al. (1993) have studied a sample of stars in the Large Magellanic Cloud (LMC), suspected to be $\mathrm{CH}$ stars, which are very luminous $\left(M_{\mathrm{bol}} \simeq-5.3\right)$. Our $\mathrm{CH}$ stars are actually much fainter $(-1.8)$. If confirmed, the existence of such bright $\mathrm{CH}$ stars should probably be explained in terms of evolutive tracks for LMC metallicity $(Z \simeq 0.008)$ shifted towards higher effective temperatures with respect to tracks for solar abundances $(Z \simeq 0.02)$. The carbon stars Azzopardi et al. (1991) and Westerlund et al. (1991) ascribed to the Galactic bulge (spheroidal region extending about $3 \mathrm{kpc}$ from the Galactic center) are finally much closer to our HC-stars. Westerlund et al. (1995) published the HR diagram of 20 bulge C-stars. Their locus corresponds to $3.53 \leq \log T_{\text {eff }} \leq 3.66$ and $-2.72 \leq \log M_{\text {bol }} \leq-1.04$, with $\left\langle T_{\text {eff }}\right\rangle \simeq 3825 \mathrm{~K}$ and $\left(M_{\mathrm{bol}}\right\rangle \simeq-1.7 \pm 0.6$ which corresponds well to the content of Table 7 for the HC-stars. The mean metallicity higher in the bulge than outside it, is possibly responsible for the mean value $\left\langle T_{\text {eff }}\right\rangle \simeq 3825 \mathrm{~K}$ slightly lower than those quoted in Table 7. Ng (1998) suggested that the stars of Azzopardi et al. are not intrinsic bulge stars, but Tyson \& Rich (1991) found that their kinematics are the same as those of bulge $\mathrm{K}$ and $\mathrm{M}$ stars. It is possible that the bulge and the thick disk are parts of the same dynamical component, at least in systems with small bulges, like the Galaxy (Freeman 1987a).

Graff et al. (2000) analyzed the velocity residuals of 551 carbon stars in the LMC. They found two different populations $\left(20 \%\right.$ of stars with $\sigma \simeq 8 \mathrm{kms}^{-1}$ and $80 \%$ with $\sigma \simeq 22 \mathrm{~km} \mathrm{~s}^{-1}$ ), and identify them with young disk and old disk populations respectively. The former, which does exhibit a slightly higher metallicity, has no counterpart in our HIPPARCOS sample. It should probably be searched for beyond the HIPPARCOS range, in the local structures of spiral arms. There is however no hot faint carbon giants in the LMC (i.e. no low-luminosity Rtype stars of our HC'-sample) which could correspond to the thick disk population. Conversely, the faint-end of the C star luminosity function is at about $M_{\text {bol }} \simeq-1.4$ in the Small Magellanic Cloud (SMC), and at $M_{\text {bol }} \simeq-1.2$ in the Fornax dwarf galaxy (Azzopardi 1999). Those numbers are in very good agreement with our mean values for early HC-stars in Table 3.

Acknowledgements. We thank Dr. Alain Jorissen who suggested part of this study. Valuable suggestions from an anonymous referee are gratefully acknowledged.

\section{Appendix A: The Sun velocity}

Introducing the galactic proper motion components

$\mu_{\mathrm{l}} \cos b=\mu_{\alpha} \cos \delta \cos \phi+\mu_{\delta} \sin \phi$

$\mu_{\mathrm{b}}=-\mu_{\alpha} \cos \delta \sin \phi+\mu_{\delta} \cos \phi$

where $\phi$ is the parallactic angle $(0 \leq \phi \leq 180)$ as given by

$\sin \phi / \cos \delta_{\mathrm{G}}= \pm \sin \left(\alpha-\alpha_{\mathrm{G}}\right) / \cos b$

where + is adopted for $\alpha>\alpha_{\mathrm{G}}$ and - for $\alpha<\alpha_{\mathrm{G}}$. The galactic coordinates are $(l, b)$, while $\left(\alpha_{\mathrm{G}}, \delta_{\mathrm{G}}\right)$ are the adopted equatorial coordinates (1950) of the North Galactic Pole G

$\alpha_{\mathrm{G}}=12 \mathrm{~h} 49.0 \mathrm{mn}=192.25 \quad \delta_{\mathrm{G}}=+27^{\circ} .4$.

The equatorial proper motion components $\mu_{\alpha} \cos \delta$ and $\mu_{\delta}$ were taken from the HIPPARCOS catalogue (ESA 1997). The velocity components are then $\left(\gamma \simeq 4.738 \mathrm{~km} \mathrm{~s}^{-1}\right)$

$U=\gamma\left[\mu_{\mathrm{l}} \cos b \sin l+\mu_{\mathrm{b}} \sin b \cos l\right] / \varpi-V_{\mathrm{r}} \cos b \cos l$

$V=\gamma\left[\mu_{\mathrm{l}} \cos b \cos l-\mu_{\mathrm{b}} \sin b \sin l\right] / \varpi+V_{\mathrm{r}} \cos b \sin l$

$W=\gamma\left[\mu_{\mathrm{b}} \cos b\right] / \varpi+V_{\mathrm{r}} \sin b$

where $\varpi$ is the true parallax as estimated in Paper I. We intend to compute the mean values $\langle U\rangle,\langle V\rangle$ and $\langle W\rangle$. With the opposite sign, this is the solar velocity relative to the considered group of stars, viz.

$u_{\odot}=-\langle U\rangle \quad v_{\odot}^{\prime}=-\langle V\rangle \quad w_{\odot}=-\langle W\rangle$

with for the mean peculiar velocity of the group

$\left\langle u_{*}\right\rangle=0 \quad\left\langle v_{*}\right\rangle=v_{\mathrm{l}} \quad\left\langle w_{*}\right\rangle=0$.

The $v_{1}$-component is not set to zero since the group may systematically lag behind the local standard of rest (LSR), and $\left\langle v_{*}\right\rangle$ averages to a negative value which is small for spiral-arm and disk stars, and large for spheroidalcomponent stars (see Mihalas \& Binney 1981, Sect. 6.4, p. 397). To derive the Sun's peculiar velocity $\left(u_{\odot}, v_{\odot}, w_{\odot}\right)$ relative to its LSR, we must determine $v_{1}$ and calculate

$v_{\odot}=-\langle V\rangle+v_{1}$

Alternatively, the solar motion may be described in terms of a speed

$S_{\odot}=\left(u_{\odot}^{2}+v_{\odot}^{\prime 2}+w_{\odot}^{2}\right)^{1 / 2}$

and the coordinates of the apex as given by

$\tan l_{\mathrm{A}}=-v_{\odot}^{\prime} / u_{\odot} \quad \sin b_{\mathrm{A}}=w_{\odot} / S_{\odot}$. 


\section{References}

Atanasijević, I. 1971, Selected exercises in galactic astronomy (D. Reidel Pub. Co., Dordrecht), 65

Azzopardi, M. 1999, Ap\&SS, 265, 291

Azzopardi, M., Rebeirot, E., Lequeux, J., \& Westerlund, B. E. 1991, A\&AS, 88, 265

Bergeat, J., \& Knapik, A. 1997, A\&A, 321, L9

Bergeat, J., Sibille, F., \& Lunel, M. 1978, A\&A, 64, 423

Bergeat, J., Knapik, A., \& Rutily, B. 1999, A\&A, 342, 773

Bergeat, J., Knapik, A., \& Rutily, B. 2001, A\&A, 369, 178

Bidelman, W. P. 1956, Vistas Astron., 2, 1428

Blanco, V. M. 1965, in Stars and Stellar Systems, Vol. 5, ed. A. Blaauw, \& M. Schmidt (Univ. of Chicago Press, Chicago), 241

Bothun, G., Elias, J. H., Mac Alpine, G., et al. 1991, AJ, 101, 2220

Buser, R., Jinxiang Rong, \& Karaali Salih 1999, A\&A, 348, 98

Busso, M., Gallino, R., \& Wasserburg, G. J. 1999, ARA\&A, 37,239

Carlberg, R. G., Dawson, P. C., Tsu, T., \& Vanderberg, D. A. 1985, ApJ, 294, 674

Charlier, C. V. L. 1926, The motion and the distribution of the stars (University of California Press, Berkeley)

Chiba, M., \& Beers, T. C. 2000, AJ, 119, 2843

Claussen, M. J., Kleinmann, S. G., Joyce, R. R., \& Jura, M. 1987, ApJS, 65, 385

Dahn, C. C., Liebert, J., Kron, R. G., Spinrad, H., \& Hintzen, P. M. 1977, ApJ, 216, 757

Dean, C. A. 1976, AJ, 81, 364

Dehnen, W. 1998, AJ, 115, 2384

Dehnen, W. 2000, AJ, 119, 800

Dehnen, W., \& Binney, J. J. 1998, MNRAS, 298, 387

Delhaye, J. 1965, in Stars and Stellar Systems, Vol. 5, ed. A. Blaauw, \& M. Schmidt (Univ. of Chicago Press, Chicago), 61

ESA 1995, Future Possibilities for Astrometry in Space, a joint RGO-ESA Workshop at Cambridge, UK, 19-21 June 1995, ESA SP-379

ESA 1997, The HIPPARCOS Catalogue, ESA SP-1200 (ESA)

Freeman, K. C. 1980, In Photometry, Kinematics and Dynamics of Galaxies, ed. D. S. Evans, University of Texas, Austin, 85

Freeman, K. C. 1987a, ARA\&A, 25, 603

Freeman, K. C. 1987b, in The Galaxy, ed. G. Gilmore, \& B. Carswell (D. Reidel Publishing Company), 291

Fuenmayor, F. J. 1981, Rev. Mex. Astron. Astrofis., 83

Gilmore, G. 1989, in The Milky Way as a Galaxy, 9th adv. Course Swiss Society of Astrophysics and Astronomy, SaasFee, ed. G. Gilmore, I. King, \& P. Van der Kruit, 369

Gilmore, G., \& Reid, N. 1983, MNRAS, 202, 1025

Gould, A., Bahcal, J. N., \& Flynn, C. 1996, ApJ, 465, 759

Graff, D. S., Gould, A. P., Suntzeff, N. B., Schommer, R. A., \& Hardy, A. 2000, ApJ, 540, 211

Green, P. J., Margon, B., \& Mac Connell, D. J. 1991, ApJ, 380, L31

Green, P. J., \& Margon, B. 1994, ApJ, 423, 723

Groenewegen, M. A. T., de Jong, T., van der Bliek, N. S., Slijkhuis, S., \& Willems, F. J. 1992, A\&A, 253, 150

Han, Z., Eggleton, P. P., Podsiadlowski, P., \& Tout, C. A. 1995, MNRAS, 277, 1443

Hanson, R. B. 1979, MNRAS, 186, 875

Hartwick, F. D. A., \& Cowley, A. P. 1985, AJ, 90, 2244

Iben, I., \& Renzini, A. 1983, ARA\&A, 21, 27
Jorissen, A., \& Boffin, H. M. J. 1992, Binaries as tracers of stellar formation (Cambridge University Press)

Keenan, P. C. 1942, ApJ, 96, 101

Keenan, P. C. 1993, PASP, 105, 905

Kerber, L. O., Javiel, S. C., \& Santiago, B. X. 2001, A\&A, 365, 424

Kerschbaum, F., \& Hron, J. 1992, A\&A, 263, 97

Kholopov, P. N., Samus, N. N., Frolov, M. S., et al. 1985, General Catalogue of Variable Stars (Nauka Publishing House), Moscow (GCVS). Suppl. Lists 67 (1985, IBVS 2681), 68 (1987, IBVS 3058), 69 (1989, IBVS 3323), 70 (1990, IBVS 3530), 71 (1993, IBVS 3840), 72 (1995, IBVS 4140), 73 (1997, IBVS 4471), 74 (1999, IBVS 4659), \& 75 (2000, IBVS 4870)

Knapik, A. 1999, Thèse, Université Claude Bernard - Lyon 1

Knapik, A., \& Bergeat, J. 1997, A\&A, 321, 236

Knapik, A., Bergeat, J., \& Rutily, B. 1998, A\&A, 334, 545 (Paper I)

Knapik, A., Bergeat, J., \& Rutily, B. 1999, A\&A, 344, 263

Knapik, A., Bergeat, J., \& Rutily, B. 2002, A\&A, submitted

Liebert, J., Schmidt, G. D., Lesser, M., et al. 1994, ApJ, 421, 733

Lutz, T. E., \& Kelker, D. H. 1973, PASP, 85, 573 (LK)

Majewski, S. R. 1993, ARA\&A, 31, 575

Malmquist, K. G. 1924, Medd. Lund Astron. Obs., Ser. II, 32, 64

Malmquist, K. G. 1936, Stockholms Obs. Medd., No. 26

Marigo, P., Girardi, L., \& Bressan, A. 1999, A\&A, 344, 123

McClure, R. D. 1997a, PASP, 109, 256

McClure, R. D. 1997b, PASP, 109, 536

McClure, R. D., Fletcher, J. M., \& Nemec, J. M. 1980, ApJ, 238, L35

McLeod, N. W. 1947, ApJ, 105, 390

Metzger, M. R., \& Schechter, P. L. 1994, ApJ, 420, 177

Mihalas, D., Binney, J. 1981, Galactic Astronomy, Structure and Kinematics, 2nd edition (Freeman \& Co, San Francisco)

Norris, J. E. 1999, Ap\&SS, 265, 213

Ng, Y. K. 1998, In Asymptotic Giant Branch, IAU Symp., 191, ed. T. Le Bertre, A. Lebre, \& C. Waelkens (Astronomical Society of the Pacific)

Ojha, D. K. 2001, MNRAS, 322, 426

Plaut, L. 1965, in Stars and Stellar Systems, Vol. 5, ed. A. Blaauw, \& M. Schmidt (Univ. of Chicago Press, Chicago), 267

Prochaska, J. X., Naumov, S. O., Carney, B. W., McWilliam, A., \& Wolfe, A. M. 2000, AJ, 120, 2513

Schwarzkopf, U., Dettmar, R.-J. 2000, A\&A, 361, 451

Smith, H. Jr., \& Eichhorn, H. 1996, MNRAS, 281, 211

Stephenson, C. B. 1989, Pub. of the Warner \& Swasey Obs., $3(2)$

Suntzeff, N. B., Phillips, M. M., Elias, J. H., et al. 1993, PASP, 105,350

Tyson, N. D., \& Rich, R. M. 1991, ApJ, 367, 547

Vandervort, G. L. 1958, AJ, 63, 311

Van Eck, S., \& Jorissen, A. 2000, A\&A, 360, 196

Wallerstein, G., \& Knapp, G. R. 1998, ARA\&A, 36, 369

Westerlund, B. E., Azzopardi, M., Breysacher, J., \& Rebeirot, E. 1991, A\&A, 244, 367

Westerlund, B. E., Azzopardi, M., Breysacher, J., \& Rebeirot, E. 1995, A\&A, 303, 107

Wielen, R., Dettbarn, C., Fuchs, B., Jahreiss, H., \& Radous, G. 1992, in The Stellar Populations of Galaxies, ed. B. Barbug, \& A. Renzini, IAU Symp., 149, 81 